\title{
Polyhedral Kähler manifolds
}

\author{
DMITRI PANOV
}

\begin{abstract}
In this article we introduce the notion of polyhedral Kähler manifolds, even dimensional polyhedral manifolds with unitary holonomy. We concentrate on the 4-dimensional case, prove that such manifolds are smooth complex surfaces and classify the singularities of the metric. The singularities form a divisor and the residues of the flat connection on the complement of the divisor give us a system of cohomological equations. A parabolic version of the Kobayshi-Hitchin correspondence of $\mathrm{T}$ Mochizuki permits us to characterize polyhedral Kähler metrics of nonnegative curvature on $\mathbb{C} P^{2}$ with singularities at complex line arrangements.
\end{abstract}

$53 \mathrm{C} 56 ; 32 \mathrm{Q} 15,53 \mathrm{C} 55$

\section{Introduction and results}

First, we recall the notion of a polyhedral metric and a polyhedral manifold and give some basic facts about them. Consider a piecewise linear connected manifold $M^{d}$ with a fixed simplicial decomposition. Let $\Delta_{i}^{d}$ be the simplices of highest dimension of this decomposition. Choose a flat metric on every $\Delta_{i}^{d}$ in such a way that every two simplices that have a common face are glued by an isometry. This gives a metric on $M^{d}$, which is called polyhedral, and $M^{d}$ is called a polyhedral manifold.

For every point $x$ of a polyhedral manifold $M^{d}$ we canonically associate its tangent cone, ie, a cone with polyhedral metric such that a neighborhood of its origin is isometric to a neighborhood of $x$. At the nonsingular points of $M^{d}$ the tangent cone is the Euclidean space $\mathbb{R}^{d}$. A polyhedral metric has no singularities at faces of codimension 1, but may have singularities at faces of codimension 2 . The tangent cone of the points in the interior of such faces is isometric to the direct product of a 2-cone and the flat space $\mathbb{R}^{d-2}$. The angle of the 2 -cone is called the conical angle at the face.

The singular locus of a polyhedral metric is naturally stratified. A point of $M^{d}$ is called a metric singularity of codimension at least $k$ if its tangent cone is not isometric to the direct product of $\mathbb{R}^{d-k+1}$ and a $(k-1)$-dimensional polyhedral cone. The set of all metric singularities of codimension at least $k$ is denoted by $M_{s}^{d-k}$. 
The complement to the singular locus of the metric is connected and we can consider the holonomy of the metric on it. This gives us a representation $\pi_{1}\left(M^{d} \backslash M_{s}^{d-2}\right) \rightarrow \mathrm{SO}(d)$ (we will consider only orientable manifolds). For a generic choice of a polyhedral metric this representation has an everywhere dense image in $\mathrm{SO}(d)$.

In this work we study even dimensional polyhedral manifolds $M^{2 n}$ whose holonomy group is contained in a subgroup of $\mathrm{SO}(2 n)$ conjugate to $U(n)$. On the complement of the singularities of the metric these manifolds have a complex structure $J$ parallel with respect to the flat metric and compatible with it. In addition to the unitarity of the holonomy we impose one condition. For any face $F$ of codimension 2 consider a simplex $\Delta^{2 n}$ that contains $F$ in its border. The parallel complex structure $J$ defined in the interior of $\Delta^{2 n}$ naturally extends to the whole $\Delta^{2 n}$ and we say that $F$ has a holomorphic direction if $F$ is a piece of a holomorphic hyperplane with respect to $J$.

Definition 1.1 A polyhedral manifold $M^{2 n}$ is called a polyhedral Kähler manifold (or shortly a PK manifold) if the holonomy of its metric belongs to a subgroup of $\mathrm{SO}(2 n)$ conjugate to $U(n)$ and every codimension 2 face with conical angle $2 k \pi$, $k \geq 2(k \in \mathbb{N})$, has a holomorphic direction.

Remark 1.2 Codimension 2 faces with conical angle different form $2 k \pi(k \in \mathbb{N})$ automatically have complex direction (cf Section 3) so we don't need to impose this condition on them. If we don't impose the condition on the faces with conical angle $2 k \pi, k \geq 2$, we obtain Thurston's $(X, G)$-cone-manifolds modeled on $X=\mathbb{C}^{n}$ with $G$ the group of unitary isometries of $\mathbb{C}^{n}[19]$.

Remark 1.3 In this work simplicial decompositions are used only to define the class of PK manifolds and play a secondary role. We will mostly think about PK manifolds as spaces with a specific metric and will not distinguish manifolds that are isometric but have different simplicial decompositions.

A 2-dimensional oriented polyhedral surface is automatically Kähler (since $\mathrm{SO}(2)=$ $U(1)$ ) and complete classification of such structures is given by Troyanov [20] (we recall this classification in Section 2). In the rest of this work we deal mostly with 4-dimensional polyhedral Kähler manifolds. In Section 2 several elementary examples of such manifolds are given.

A polyhedral metric is called nonnegatively curved if the conical angle at every singular face of codimension 2 is smaller than $2 \pi$. The original motivation for our study of PK metrics is due to the following remark of Anton Petrunin. The holonomy of a nonnegatively curved polyhedral $\mathbb{C} P^{n}$ preserves a symplectic form (see a related 
discussion in Petrunin [17]). This is a partial case of a vanishing theorem proved by Cheeger [2]. This means that a nonnegatively curved $\mathbb{C} P^{n}$ is PK; we discuss this subject in Section 2.

Known examples An explicit example of a nonnegatively curved polyhedral $\mathbb{C} P^{2}$ is provided by Kühnel's 9 vertices triangulation [10]. This polyhedral $\mathbb{C} P^{2}$ can be obtained as a finite isometric quotient of a flat complex 2-torus, and the holonomy of the metric on $\mathbb{C} P^{2}$ is finite. In general for any $n$ there exist a series of polyhedral metrics on $\mathbb{C} P^{n}$ that are obtained as quotients of complex tori; see Kaneko, Tokunaga and Yoshida [9].

Couwenberg, Heckman and Looijenga [3] study geometric structures that are more general than PK metrics. They obtain constant holomorphic curvature metrics on $\mathbb{C} P^{n}$, having as singular locus complex reflection hyperplane arrangements. Their approach is different; in particular from the very beginning they start with a holomorphic manifold. It should be possible to prove that in the case when curvature is zero their metrics are PK.

Acknowledgments This article contains a revised part of my $\mathrm{PhD}$ written at École Polythechnique under supervision of Maxim Kontsevich and defended in 2005. I am very indebted to Maxim for his insight, inspiration, guidance and help during my $\mathrm{PhD}$; many of his ideas are contained in this work. The remark of Anton Petrunin started this research and Misha Gromov gave me the first example of a PK metric on $\mathbb{C} P^{2}$. Discussions with Dima Zvonkine and Christophe Margerin helped to go further, and the explanation of Takuro Mochizuki of some of his results permitted me to finish this work. I would also like to thank Eduard Looijenga, Nikita Markarian, Carlos Simpson, Misha Verbitski and Jean Yves Welschinger. Without discussions, comments and encouragement of all these people this article would not be written. Finally I would like to thank members of the geometry group at Imperial for the excellent environment. This work is supported by EPSRC grant EP/E044859/1.

\subsection{Local properties of PK metrics}

A PK manifold has a natural complex structure defined outside the singular locus; it is constant in the local flat coordinates. We will prove that for a 4-dimensional PK manifold $M^{4}$ this complex structure can be extended to the whole manifold.

Definition 1.4 Let $M^{4}$ be a PK manifold. Holomorphic functions on $M^{4}$ are defined as continuous functions on $M^{4}$ that are holomorphic on the complement to the singularities $M^{4} \backslash M_{S}^{2}$. 
A holomorphic chart in $M^{4}$ is an open subset $U$ with an injective map $\varphi=(f, g): U \rightarrow$ $\mathbb{C}^{2}$ with $f$ and $g$ holomorphic as above and such that $\varphi\left(M_{s}^{2} \cap U\right)$ is an analytic subset of $\varphi(U) \subset \mathbb{C}^{2}$.

The following theorem justifies this definition by proving that holomorphic functions and holomorphic charts on $M^{4}$ define together a genuine complex structure on $M^{4}$.

Theorem 1.5 Every point of a 4-dimensional PK manifold is contained in a holomorphic chart. Holomorphic charts form together a holomorphic atlas on $M^{4}$ and induce on it a structure of a smooth complex surface. The singular set $M_{S}^{2}$ is a complex curve for the defined holomorphic structure.

Remark 1.6 We don't consider any intermediate smooth structure on $M^{4}$ in order to define a complex structure on it. At the same time, it is known that PL manifolds of dimension up to 6 have a canonical smooth structure.

The main step in Theorem 1.5 is the construction of holomorphic charts for the singularities of the PK metric. A neighborhood of every singularity embeds isometrically into its tangent cone, and it is possible to introduce on the tangent cone the structure of a single holomorphic chart. We call these cones polyhedral Kähler cones, and denote by $C_{K}^{4}$. All PK cones have a natural holomorphic Euler vector field e (cf Section 3.1) that acts by dilatations of the metric, and this field is crucial for us.

Theorem 1.7 Let $C_{K}^{4}$ be a 4-dimensional polyhedral Kähler cone. There exists a homeomorphism $\varphi: C_{K}^{4} \rightarrow \mathbb{C}^{2}$ holomorphic outside the singularities of the cone and satisfying the following property: The Euler field $e$ written in coordinates $(z, w)$ of $\mathbb{C}^{2}$ is given by

$$
\frac{1}{\alpha} z \frac{\partial}{\partial z}+\frac{1}{\beta} w \frac{\partial}{\partial w}
$$

where $\alpha$ and $\beta$ are positive real numbers. The image of the singular locus of $C_{K}^{4}$ under the map $\varphi$ is given by a union of curves $c_{1} z^{\alpha}=c_{2} w^{\beta}$.

The singularity is called irrational if $\alpha / \beta \in \mathbb{R} \backslash \mathbb{Q}$. In this case its tangent cone is isometric to the direct product of two 2 -cones $C_{1} \times C_{2}$ with conical angles $2 \pi \alpha$ and $2 \pi \beta$.

The singularity is called rational of type $(p, q, \alpha), p, q \in \mathbb{N}$ if its Euler field is equal to $e=(p / \alpha) z(\partial / \partial z)+(q / \alpha) w(\partial / \partial w)$ in coordinates $(z, w)$. Here $p$ and $q$ are relatively prime, $p \leq q$ and $\alpha$ is a positive real number. Sometimes, when the choice of $\alpha$ is not important, we may also say that the singularity is of type $(p, q)$. Since $e$ 
acts by dilatation of the metric, it preserves the singular locus of the metric. Thus, in the neighborhood of $x$ any irreducible component of the singular locus is a curve given by one of the equations: $c z^{q}=w^{p}, c \neq 0 ; z=0$; or $w=0$. All these curves are flat with respect to the induced PK metric. Each curve $c z^{q}=w^{p}$ has a conical point at the origin with the same angle $2 \pi \alpha$, the line $z=0$ has conical angle $(2 \pi / p) \alpha$, and the line $w=0$ has conical angle $(2 \pi / q) \alpha$.

The next theorem gives a description of the set $S(p, q, \alpha)$ of equivalence classes of singularities of type $(p, q, \alpha)$. There is a slight difference between the cases $(1=p=q)$, $(1=p<q),(1<p<q)$. The triple $(p, q, \alpha)$ does not determine the singularity uniquely, the singularities of a given type form an infinite-dimensional space.

Theorem 1.8 On a 2-sphere, consider the set of metrics (up to isometry) of curvature 4 , with area $\pi \alpha /(p q)$ and having an arbitrary number of conical points. Moreover if $p>1$ and $q>1$ we mark two conical points, while if $p=1, q>1$ we mark one conical point. This set of metrics on the sphere is in natural 1-to-1 correspondence with the set $S(p, q, \alpha)$.

Consider a $(p, q, \alpha)$ singularity, let $\left(2 \pi \beta_{1}, \ldots, 2 \pi \beta_{n}\right)$ be the conical angles of the PK metric at the singular branches $c_{k} z^{q}=w^{p},\left(c_{k} \neq 0\right)$. Let $2 \pi \beta_{z}$ be the angle at $z=0$ and $2 \pi \beta_{w}$ the angle at $w=0$.

Theorem 1.9 The following relation holds:

$$
\alpha=\frac{p q}{2}\left(\sum_{k}\left(\beta_{k}-1\right)+\frac{\beta_{z}-1}{p}+\frac{\beta_{w}-1}{q}\right)+\frac{p+q}{2} .
$$

Theorems 1.5-1.9 are proven in Section 3.

\subsection{Flat connection and topological relations}

By Theorem 1.5 every 4-dimensional PK manifold is a complex surface and the singular locus of the PK metric forms a complex curve on it. Further on will denote the surface by $S$ and the complex curve by $\Gamma$.

The PK metric on $S$ defines a flat meromorphic connection on the tangent bundle of $S$ with first order poles at $\Gamma$. In Section 4 we study this connection, especially in the neighborhood of singularities of complex codimension 2 . We give a list of conditions that imply that a connection on the tangent bundle to a surface is a connection of a PK metric (Theorem 4.13). Using the residues of the connection we write down a system of topological relations on the pair $(S, \Gamma)$. This is done in Section 5 . 
Let us fix some notation. Irreducible components of $\Gamma$ will be denoted by $\Gamma_{j}$. For every component $\Gamma_{j}$ we denote by $2 \pi \beta_{j}$ the conical angle at $\Gamma_{j}$, ie, the angle of a 2 -cone orthogonal to any nonsingular point of $\Gamma_{j}$. The singularities of $\Gamma$ that are not normal crossings are denoted by $x_{i}$ and their type is denoted by $\left(p_{i}, q_{i}, \alpha_{i}\right)$.

Definition 1.10 For any surface $S$ a collection of divisors $\Gamma_{j}$ with positive weights $\beta_{j}$ is called a weighted arrangements. In the case when there exists a PK metric on $S$ with singularities at $\Gamma_{j}$ of angles $2 \pi \beta_{j}$ we call $\left(\Gamma_{j}, \beta_{j}\right)$ the weighted arrangement of the PK metric or the PK arrangement. Sometimes we will mean by weighted arrangement the whole data $\left(\Gamma_{j}, \beta_{j} ; x_{i}, p_{i}, q_{i}, \alpha_{i}\right)$.

Define two numbers related to the behavior of $\Gamma_{j}$ in the neighborhood of $x_{i}$. Denote by $\tilde{d}_{i j}$ the number of branches (local irreducible components) of $\Gamma_{j}$ at $x_{i}$. Additionally let $d_{i j}$ be the number of branches, except counting branches $z=0$ and $w=0$ with weights $1 / p$ and $1 / q$. Denote by $B_{j k}, j \neq k$, the number of intersections of curves $\Gamma_{j}$ and $\Gamma_{k}$ that represent the normal crossing singularity of $\Gamma$ and define $B_{j j}$ by

$$
B_{j j}=-\Gamma_{j} \cdot \Gamma_{j}+\sum_{i} p_{i} q_{i}\left(d_{i j}\right)^{2}
$$

where $\Gamma_{j} \cdot \Gamma_{j}$ is the self-intersection number of $\Gamma_{j}$.

Theorem 1.11 Any weighted arrangement $\left(\Gamma_{j}, \beta_{j} ; x_{i}, p_{i}, q_{i}, \alpha_{i}\right)$ of a PK metric satisfies the following relations:

$$
\begin{aligned}
\forall j \quad \sum_{k} B_{j k}\left(\beta_{k}-1\right) & =-2 \chi\left(\Gamma_{j}\right)-K_{S} \cdot \Gamma_{j}-\sum_{i}\left(d_{i j}\left(p_{i}+q_{i}\right)-2 \tilde{d}_{i j}\right), \\
-c_{1}(T S) & =K_{S}=\sum_{j}\left(\beta_{j}-1\right)\left[\Gamma_{j}\right] \in H_{2}(S, \mathbb{R}) .
\end{aligned}
$$

Moreover, in the case when for every $i \quad p_{i}=q_{i}=1$ we have the following expression for the second Chern class:

$$
c_{2}(T S)=\sum_{i}\left(\alpha_{i}-1\right)^{2}+\sum_{j \neq k} \frac{1}{2} B_{j k}\left(\beta_{j}-1\right)\left(\beta_{k}-1\right) .
$$

Here $K_{S}$ is the canonical class of $S$ and $\chi\left(\Gamma_{j}\right)$ is the Euler characteristic of the normalization of $\Gamma_{j}$.

These relations have the following nature. Relation (1-1) is a consequence of the Gauss-Bonnet formula applied to the curve $\Gamma_{j}$. For every $\Gamma_{j}$ the sum of the defects of its conical points is equal to its Euler characteristic. Relations (1-2) and (1-3) 
express the Chern classes of $T S$ in terms of the residues of the flat connection on $T S$ corresponding to the PK metric; see Ohtsuki [15].

Construction of weighted arrangements satisfying equations of Theorem 1.11 is a problem of independent interest, leading to questions of the following type:

Problem Classify arrangements of $3 n$ lines on $\mathbb{C} P^{2}$ such that every line intersect other lines exactly at $n+1$ points.

It is easy to see that such line arrangements with weights $\beta_{j}=(n-1) / n$ satisfy Equations (1-1), (1-2) and (1-3). This problem appeared previously in Hirzebruch [7] and a list of two infinite series and several exceptional arrangements satisfying the condition was given (all these arrangements are complex reflection arrangements). Further questions of similar nature (about simplicial and limit PK arrangements with a cusp) are formulated in Section 5.

\subsection{Reconstruction of nonnegatively curved PK metrics from weighted arrangements}

One of the main results of this paper is the following theorem.

Theorem 1.12 Consider a weighted arrangements of lines $\left(L_{j}, \beta_{j}\right)$ in $\mathbb{C} P^{2}$ satisfying the following conditions:

$$
\sum_{j}\left(\beta_{j}-1\right)=-3, \quad 0<\beta_{j}<1, \quad \sum_{j} d_{i j}\left(\beta_{j}-1\right)>-2 .
$$

Then the following inequality holds:

$$
\sum_{i}\left(\alpha_{i}-1\right)^{2}-\sum_{j} \frac{1}{2}\left(1-\beta_{j}\right)^{2} B_{j j}-\frac{3}{2} \leq 0 .
$$

Moreover, if the equality holds then there exists a PK metric on $\mathbb{C} P^{2}$ with conical angles $2 \pi \beta_{j}$ at $L_{j}$ (ie, $\left(L_{j}, \beta_{j}\right)$ is a $\mathrm{PK}$ arrangement.)

We prove this theorem in Section 7 after recalling (Section 6) the technique of parabolic bundles. To every arrangement that satisfies condition of the theorem we associate a parabolic structure on the pullback of the tangent bundle of $\mathbb{C} P^{2}$ to the blow up of $\mathbb{C} P^{2}$ at the multiple points of the arrangement. We prove that constructed parabolic bundle is stable and calculate its parabolic Chern characters. Inequality (1-5) is a consequence of Bogomolov-Gieseker inequality [13] (see also [12]). The existence of a PK metric in the case of equality follows form the parabolic version of Kobayashi-Hitchin correspondence from [13] and additional statements about logarithmic connection the we prove in Section 4. 


\section{Examples of polyhedral Kähler manifolds}

In this section we recall the classification of PK structures on complex curves in Troyanov [20] and give several examples of polyhedral Kähler manifolds of higher dimension.

\subsection{Flat metrics on surfaces}

Structures on a 2-dimensional polyhedral cone A 2-dimensional polyhedral cone is a very simple object but already it supports the majority of geometric structures that are essential for this work. Let us describe these structures. Consider a 2-cone $C^{2}$ with conical angle $2 \pi \alpha$. Note first that the flat metric on $C^{2} \backslash 0$ defines a conformal and hence a holomorphic structure on $C^{2} \backslash 0$. Moreover $C^{2} \backslash 0$ is biholomorphic to $\mathbb{C}^{*}$, so we can chose a holomorphic coordinate $z$ on it (defined up to a multiplicative constant). This coordinate can be used to extend the complex structure from $C^{2} \backslash 0$ to $C^{2}$. We have a natural action of $\mathbb{R}^{*}$ on $C^{2}$ by homotheties, corresponding vector field can be complexified and we call it Euler field. This field is given in the coordinate $z$ by the formula $(z / \alpha)(\partial / \partial z)$, the imaginary part of the field acts by isometries of the cone. The metric induces a flat meromorphic connection on the tangent bundle to the cone and it is given by $\nabla=d+(\alpha-1)(d z / z)$. The multivalued flat coordinate on the cone, ie, a coordinate in which the connection on $C^{2}$ is trivial, is given by $z^{\alpha}$.

The next theorem classifies polyhedral metrics on surfaces.

Theorem 2.1 (Troyanov [20]) Consider a complex curve $\Gamma$ of genus $g$ with pairwise distinct marked points $x_{1}, \ldots, x_{n}$. Let $\alpha_{1}, \ldots, \alpha_{n}$ be real positive numbers such that $\sum\left(\alpha_{i}-1\right)=2 g-2$. Then there is a unique (up to a real multiplication constant) complete flat metric on $\Gamma$ with conical points of angles $2 \pi \alpha_{i}$ at $x_{i}$ whose conformal structure on $\Gamma \backslash\left\{x_{1}, \ldots, x_{n}\right\}$ is the same as of $\Gamma$ itself.

For completeness we give here a proof of the theorem.

Lemma 2.2 For every real $\beta_{1}, \ldots, \beta_{n}$ such that $\sum_{i} \beta_{i}=0$, there exists a unique meromorphic 1-form $\eta$ on $\Gamma$ with simple poles with residues $\beta_{1}, \ldots, \beta_{n}$ at the points $x_{1}, \ldots, x_{n}$, having purely imaginary periods (ie, for every closed path $\gamma \in \Gamma$ we have $\left.\int_{\gamma} \eta \in i \mathbb{R}\right)$.

Proof By Dirichlet's theorem there exists a unique (up to a constant) real harmonic function $f$ on $\Gamma$, satisfying the equation $\Delta f=\sum_{i} \beta_{i} \delta_{x_{i}}$. This function has logarithmic poles at $x_{1}, \ldots, x_{n}$. The 1 -form $\eta$ is then given by $\eta(\vec{u})=d f(\vec{u})+i d f(J \vec{u})$, where $J$ defines the complex structure on $T \Gamma$. 
Proof of Theorem 2.1 Existence Let $\xi$ be a holomorphic differential on $\Gamma$ with simple zeros $y_{1}, \ldots, y_{2 g-2}$. It defines a flat metric $\xi \otimes \bar{\xi}$ on $\Gamma$ with conical points of angle $4 \pi$ at the points $y_{i}$. Denote by $\nabla$ the corresponding connection. Consider the 1-form $\eta$ on $\Gamma$ with purely imaginary periods that has residue -1 at any point $y_{i}$ and residue $\left(\alpha_{j}-1\right)$ at any point $x_{j}$.

Let us prove that the connection $\nabla+\eta$ on $\Gamma$ is unitary. Indeed, the holonomy of $\nabla+\eta$ along a closed path $\gamma$ is given by the formula

$$
\operatorname{hol}_{\gamma}(\nabla+\eta)=\operatorname{hol}_{\gamma}(\nabla) \exp \left(-\int_{\gamma} \eta\right)=\exp \left(-\int_{\gamma} \eta\right) .
$$

The first equality follows from the definition of holonomy and the second follows from the fact that the holonomy of $\nabla$ is trivial. The connection $\nabla+\eta$ defines a unique (up to a real multiplication constant) flat metric on $\Gamma$. In order to define it one should fix the metric at any point of $\Gamma$ different from $x_{j}$ and translate it by means of $\nabla+\eta$ to other points of $\Gamma$. The metric constructed this way has singularities exactly at $x_{j}$, and the conical angles at $x_{j}$ are defined by the poles of $\eta$.

Uniqueness Suppose that we have two metrics $g_{1}$ and $g_{2}$ satisfying the conditions of the theorem. Then the 1 -form $\nabla_{g_{1}}-\nabla_{g_{2}}$ should be holomorphic and it should have purely imaginary periods (since both $\nabla_{g_{1}}$ and $\nabla_{g_{2}}$ are unitary), ie, it is identically zero. Thus $g_{1}$ and $g_{2}$ coincide.

\subsection{Polyhedral Kähler manifolds of higher dimension}

Recall that a polyhedral manifold is called nonnegatively curved if the conical angles at all faces of codimension 2 are at most $2 \pi$.

Proposition 2.3 Let $M^{2 n}$ be a nonnegatively curved manifold that has a second cohomology class $h \in H^{2}\left(M^{2 n}\right)$ such that $h^{n}$ is non zero in $H^{2 n}\left(M^{2 n}\right)$. Then the holonomy of $M^{2 n}$ is contained in $U(n)$, ie, such a manifold is PK.

This proposition is a simple corollary of results of J Cheeger [2], which we will now describe. We don't need these results in full generality; instead, we give a version sufficient for our considerations.

Let $M^{n}$ be a polyhedral manifold, and let $M_{s}^{n-2}$ be the subset of all its metric singularities. Denote by $H_{L_{2}}^{i}\left(M^{n}\right)$ the space of $L_{2}$-harmonic forms on $M^{n} \backslash M_{s}^{n-2}$ that are closed and coclosed.

Theorem A $\operatorname{dim}\left(H_{L_{2}}^{i}\left(M^{n}\right)\right)=b^{i}\left(M^{n}\right)$.

Theorem B Suppose that the manifold $M^{n}$ is nonnegatively curved. Then every harmonic form $h$ in $H^{i}\left(M^{n}\right)$ is parallel, ie, $\nabla h=0$. 
Remark 2.4 According to [2], Theorem B indicates that nonnegatively curved polyhedral manifolds are analogs of smooth Riemannian manifolds with nonnegative curvature operator (rather then smooth manifolds with nonnegative sectional curvature). In fact, Petrunin [17] proved that analogues curvature inequality in the smooth case is even stronger.

Let us deduce Proposition 2.3 from Theorems A and B. We need a simple fact from linear algebra.

Lemma 2.5 Consider an Euclidean space $V^{2 n}$ with a nondegenerate 2-form $w$, $w^{n} \neq 0$. Denote by $S_{w}$ the subgroup of $\mathrm{SO}(2 n)$ that preserves $w$. Then the group $S_{w}$ is contained in a subgroup of $\mathrm{SO}(2 n)$ conjugate to $U(n)$.

Proof Take orthonormal coordinates $\left(x_{i}, y_{i}\right)$ in $V^{2 n}$ such that $w=\sum_{i} a_{i} d x_{i} \wedge d y_{i}$ $\left(a_{i} \neq 0\right)$. It is easy to see that every element of $\mathrm{SO}(2 n)$ that preserves $w$ preserves the form $w^{\prime}=\sum_{i} d x_{i} \wedge d y_{i}$. The stabilizer of $w^{\prime}$ in $\operatorname{SO}(2 n)$ is exactly $U(n)$.

Proof of Proposition 2.3 Let $g$ be a nonnegatively curved polyhedral metric on $M^{2 n}$. By Theorem A there exists a harmonic 2-form $w$ on $M^{2 n}$ such that $\int_{M} w^{n} \neq 0$. By Theorem B $w$ parallel in the flat metric. It has constant rank outside of the singularities, and since $\int_{M} w^{n} \neq 0, w$ should be nondegenerate. The holonomy of $g$ preserves $w$, thus by Lemma 2.5 the holonomy is contained in a subgroup of $\mathrm{SO}(2 n)$ conjugate to $U(n)$, ie, $\mathrm{g}$ is a polyhedral Kähler metric.

This proposition indicates that it should be difficult to construct an explicit simplicial decomposition on $\mathbb{C} P^{n}$ that defines a nonnegatively curved metric. All examples of PK metrics on $\mathbb{C} P^{n}$ that we know come from algebraic geometry, and produce a metric without a chosen simplicial decomposition.

\section{Examples of nonnegatively curved polyhedral $\mathbb{C} P^{n}$}

Example 1 Choose any nonnegatively curved polyhedral metric on $\mathbb{C} P^{1}$. Consider the $n$-th symmetric power $\operatorname{Sym}^{\mathrm{n}}\left(\mathbb{C} P^{1}\right)$ of $\mathbb{C} P^{1}$ with induced polyhedral metric. We have $\operatorname{Sym}^{n}\left(\mathbb{C} P^{1}\right) \simeq \mathbb{C} P^{n}$, and it is clear that the constructed polyhedral metric on $\mathbb{C} P^{n}$ is nonnegatively curved. This is the first nontrivial example of a higher-dimensional PK manifold that I learned and it was proposed to me by M Gromov.

For $n=2$ we obtain a PK metric on $\mathbb{C} P^{2}$ with singularities at a conic and several lines tangent to it. The conical angle at the conic is equal to $\pi$ and the sum of defects of the conical angles at the lines is equal to $-4 \pi$. The conic is the image of the diagonal of $\mathbb{C} P^{1} \times \mathbb{C} P^{1}$. 
Example 2 Let $T^{2}$ be a real 2 -torus with a flat metric. Consider the $(n+1)$-th power of $T^{2}, T^{2 n+2}=\left(T^{2}\right)^{n+1}$. Let $T^{2 n}$ be a subtorus of $T^{2 n+2}$ given by the equation $\sum_{i} x_{i}=0, x_{1}, \ldots, x_{n+1} \in T^{2}$. Let $S_{n+1}$ be the permutation group acting on $T^{2 n+2}$.

Lemma 2.6 The quotient $T^{2 n} / S_{n+1}$ is a $\mathbb{C} P^{n}$ with a nonnegatively curved polyhedral metric.

Proof Let $E$ be the unique elliptic curve with the same conformal structure as $T^{2}$. Let $L_{n}$ be a complex line bundle over $E$ with first Chern class $n+1$. Then $T^{2 n} / S_{n+1}$ can be identified with the space of zero divisors of sections of $L_{n}$.

For $n=2$ we obtain a PK metric on $\mathbb{C} P^{2}$ singular along an elliptic curve of degree 6 with 9 cusps. This curve is projectively dual to a smooth cubic.

Remark 2.7 It follows from Theorem B that an orientable 4-dimensional manifold admitting a nonnegatively curved polyhedral metric with irreducible holonomy is homeomorphic to $S^{4}$ or $\mathbb{C} P^{2}$ (recall that irreducible means no invariant subspaces). Nonnegative polyhedral metrics on $S^{2} \times S^{2}$ were studied by Orshanskiy [16] using the theory of Alexandrov spaces. The results of that paper can be obtained in a different way using complex geometry.

PK metrics via branched covering One can construct polyhedral Kähler metrics via branched coverings. Let $f: S_{2} \rightarrow S_{1}$ be a branched covering of a smooth complex surface $S_{1}$ by a smooth complex surface $S_{2}$. Suppose that $S_{1}$ has a polyhedral Kähler metric and $f$ is ramified over a set of flat curves on $S_{1}$. Then the pullback of the metric on $S_{2}$ is a polyhedral Kähler metric.

Consider the map $f: \mathbb{C} P^{2} \rightarrow \mathbb{C} P^{2}, f(x: y: z)=\left(x^{n}: y^{n}: z^{n}\right)$. This map is ramified at the lines $x=0, y=0, z=0$. The following two examples use this map to produce new PK metrics.

Example 3 ( 7 lines) Consider a PK metric on $\mathbb{C} P^{2}$ with the singular locus given by the lines $x=0, y=0, z=0$ and a conic tangent to these lines (cf Example 1). The conical angle at the conic is $\pi$ and the conical angles at the lines are equal to $2 \pi \alpha$, $2 \pi \beta, 2 \pi \gamma, \alpha+\beta+\gamma=1$. Consider the branched covering $f(x: y: z)=\left(x^{2}: y^{2}: z^{2}\right)$. Then the singular locus of the pullback metric is composed of 7 lines, 4 of which have conical angle $\pi$ and three of which have angles $4 \pi \alpha, 4 \pi \beta, 4 \pi \gamma$. 
Example 4 (A metric on a symmetric $K 3$ surface) Consider a PK metric on $\mathbb{C} P^{2}$ with the singular locus given by the lines $x=0, y=0, z=0, x+y=z$ (all of them having conical angle $\pi$ ) and a conic tangent to these lines (Example 1). Consider the pullback metric on $\mathbb{C} P^{2}$ under the map $f(x: y: z)=\left(x^{6}: y^{6}: z^{6}\right)$. The preimage of the line $x+y=z$ is given by the equation $x^{6}+y^{6}=z^{6}$. The double cover of $\mathbb{C} P^{2}$ ramified over the curve $x^{6}+y^{6}=z^{6}$ is a $K 3$ surface. This construction gives a polyhedral Kähler metric on it.

Example 5 (PK metrics on algebraic Kummer surfaces) Recall that a Kummer $K 3$ surface is obtained from a complex 2-torus $T^{2}$ by the quotient with respect to the involution $I: x \rightarrow-x$ and successive blow up of 16 fixed points. If we first blow up the points on $T^{2}$ fixed by $I$, we get a surface that is a double cover of the Kummer surface. So in order to get a PK metric on a Kummer surface it will be sufficient to construct any $I$-invariant PK metric on $T^{2}$ blown up at 16 invariant points.

Let $\Gamma$ be a genus 2 curve, $\sigma$ its hyperelliptic involution, and $\operatorname{Jac}_{2}(\Gamma)$ the Jacobian of degree 2 line bundles on $\Gamma$. Let $g$ be a flat metric with conical points on $\Gamma$, invariant under $\sigma$ (we suppose that the conformal structure of $g$ is that of $\Gamma$ ). The metric $g$ induces a PK metric $\widetilde{g}$ on the symmetric square $\operatorname{Sym}^{2}(\Gamma)$ of $\Gamma$.

Recall that $\operatorname{Sym}^{2}(\Gamma)$ is naturally isomorphic to the blow up of $\operatorname{Jac}_{2}(\Gamma)$ at the point corresponding to the canonical class of $\Gamma$. Moreover the involution $\sigma$ on $\Gamma$ induces the involution $I$ on the blown up of $\operatorname{Jac}_{2}(\Gamma)$. Consider the degree 16 cover of $\operatorname{Sym}^{2}(\Gamma)$ corresponding to the subgroup $(2 \mathbb{Z})^{4} \subset H_{1}\left(\operatorname{Sym}^{2}(\Gamma)\right)$. One can check that the involution $I$ lifts to this cover and it fixes 16 exceptional curves. Moreover, $I$ fixes the lift of $\widetilde{g}$. This finishes the construction.

Nonelementary examples All the examples of PK manifolds listed above are obtained by relatively elementary constructions. One of the main goals of this paper is to construct PK metrics on $\mathbb{C} P^{2}$ with singularities at lines arrangements, and some examples of such arrangements are given in Section 5.3. To construct the corresponding metric on $\mathbb{C} P^{2}$ we need to use deep results of T Mochizuki.

\section{Singularities of 4-dimensional PK manifolds}

Starting from this section we deal only with 4-dimensional PK manifolds. In the next two subsections we will prove Theorems 1.5 and 1.7. Before doing this let us explain why existence of holomorphic charts (Definition 1.4) on a polyhedral Kähler manifold $M^{4}$ implies immediately that $M^{4}$ is a complex surface. 
Indeed, suppose that $M^{4}$ can be covered by holomorphic charts $\left(U_{\alpha}, \varphi_{\alpha}\right)$. To prove that $\left(U_{\alpha}, \varphi_{\alpha}\right)$ is a holomorphic atlas on $M^{4}$ we need to show that for every $\alpha$ and $\beta$ the gluing map

$$
\varphi_{\alpha} \varphi_{\beta}^{-1}: \varphi_{\beta}\left(U_{\alpha} \cap U_{\beta}\right) \rightarrow \varphi_{\alpha}\left(U_{\alpha} \cap U_{\beta}\right)
$$

is holomorphic. By Definition 1.4 the map $\varphi_{\alpha} \varphi_{\beta}^{-1}$ is continuous on $\varphi_{\beta}\left(U_{\alpha} \cap U_{\beta}\right)$ and holomorphic on the complement to an analytic subset. So by standard results it is holomorphic on the whole domain $\varphi_{\beta}\left(U_{\alpha} \cap U_{\beta}\right)$.

The same argument gives us the following lemma.

Lemma 3.1 For $i=0,1$ suppose that every point of $M^{4} \backslash M_{s}^{i}$ has a holomorphic chart. Then the space $M^{4} \backslash M_{s}^{i}$ has a well-defined holomorphic structure.

The proof of Theorem 1.5 will be done in 3 steps. First we show that every point in $M_{s}^{2} \backslash M_{s}^{1}$ is contained in a holomorphic chart. Then we prove that singularities of pure codimension 3 don't exit, ie, $M_{s}^{1}=M_{s}^{0}$. And finally for singularities of codimension 4 the existence of a holomorphic chart is claimed by Theorem 1.7.

\subsection{Complex structure in codimension 4 and the Euler field}

Lemma 3.2 Every point $x \in M_{s}^{2} \backslash M_{s}^{1}$ is contained in a holomorphic chart. In particular the space $M^{4} \backslash M_{s}^{1}$ has a well-defined holomorphic structure.

Proof It is sufficient to prove this lemma for tangent cones of points in $M_{s}^{2} \backslash M_{S}^{1}$, ie for PK manifolds that are direct products of a 2-cone $C^{2}$ and the Euclidean plane $\mathbb{R}^{2}$.

By Definition 1.1 the complex structure on $\mathbb{R}^{2} \times\left(C^{2} \backslash 0\right)$ is constant with respect to the flat connection of the metric and invariant with respect to the holonomy around $\left(\mathbb{R}^{2}, 0\right)$. If the conical angle $2 \pi \beta$ of $C^{2}$ is not divisible by $2 \pi$ then this holonomy is nontrivial, it rotates the tangent planes of the horizontal fibers $\left(*, C^{2} \backslash 0\right)$ by the angle $2 \pi\{\beta\}$. So these fibers are holomorphic with respect to the complex structure. The fibers $\left(\mathbb{R}^{2}, *\right)$ are orthogonal to $\left(*, C^{2} \backslash 0\right)$ and so they are holomorphic, since $J$ preserves the metric. Thus the complex structure on $M^{4}=\mathbb{R}^{2} \times\left(C^{2} \backslash 0\right)$ is given by the product of the natural complex structures on $\mathbb{R}^{2}$ and $C^{2} \backslash 0$. Finally we note that $C^{2} \backslash 0$ is biholomorphic to $\mathbb{C}^{*}$, so there is a coordinate $z$ on $C^{2}$ holomorphic on $C^{2} \backslash 0$ and continuous on $C^{2}$. The coordinate $z$ together with a holomorphic coordinate $w$ on $\mathbb{R}^{2}$ define the structure of a chart on $\mathbb{R}^{2} \times C^{2}$. The existence of the complex structure on $M^{4} \backslash M_{s}^{1}$ follows now from Lemma 3.1. The case $2 \pi \beta=2 \pi k$, $k \geq 2$ is similar, the holonomy is trivial this time but by Definition 1.1 the vertical fiber $\mathbb{R}^{2} \times 0$ has a holomorphic direction. 
Remark 3.3 We proved that every 2-face of a 4 dimensional polyhedral Kähler manifold that belongs to the singular locus has a holomorphic direction. We need to impose the condition on the faces with conical angle $2 k \pi, k \geq 2$, in order to be able to extend the complex structure on these faces. Indeed, for a degree $k$ ramified cover of $\mathbb{C}^{2}$ with a branching of order $k$ over a totally real two-dimensional plane, the complex structure on the cover can not be extended on the branching locus.

Definition 3.4 Let $M^{4}$ be a PK manifold and let $U$ be the universal cover of $M^{4} \backslash M_{s}^{2}$. The enveloping map $E$ of $M^{4}$ is defined as a locally isometric map $E: U \rightarrow \mathbb{C}^{2}$. Equivalently this map can be seen as a multivalued map from $M^{4}$ to $\mathbb{C}^{2}$ that is locally isometric outside of $M_{s}^{2}$ and has infinite ramification at $M_{s}^{2}$. The image of $M_{s}^{2}$ under the map is called branching set $B(E)$ of $E$, it is composed of linear holomorphic faces. Note that $B(E)$ is usually everywhere dense in $\mathbb{C}^{2}$ but in the case when $B(E)$ is closed the restriction map $E: E^{-1}\left(\mathbb{C}^{2} \backslash B(E)\right) \rightarrow \mathbb{C}^{2} \backslash B(E)$ is a covering map.

Proposition 3.5 Any PK cone $C_{K}^{4}$ that is a product of $\mathbb{R}$ with a 3-cone is isometric to the product of $\mathbb{C}$ with a 2-cone. So 4-dimensional PK manifolds can not have singularities of pure codimension 3 .

Proof Suppose that $C_{K}^{4}$ is isometric to $\mathbb{R} \times P^{3}$. Denote by $v$ the constant vector field on $C_{K}^{4}$ tangent to the vertical lines $(\mathbb{R}, *)$. This field is acting on $\mathbb{R} \times\left(P^{3} \backslash 0\right)$ preserving the complex structure defined by Lemma 3.2. Consider the field $J(v)$ obtained from $v$ by the complex rotation and let $v_{\mathbb{C}}=v+i J(v)$ be the complexification of $v$. The field $v_{\mathbb{C}}$ is constant in the flat holomorphic coordinates on the complement to the singularities. Moreover, since the singularities of $C_{K}^{4}$ are tangent to $v$ and they are holomorphic on $\mathbb{R} \times\left(P^{3} \backslash 0\right), v_{\mathbb{C}}$ is also tangent to the singularities.

Consider now the enveloping map $E$ of $C_{K}^{4}$ and let us show that its branching locus is contained in a complex line through $E(0)$ (note that the image of the center 0 of $C_{K}^{4}$ is well defined). Indeed, the singularities of $C_{K}^{4}$ are of the form $\mathbb{R} \times r_{i}$, where $r_{i}$ is a singular ray of $P^{3}$. The image of $\mathbb{R} \times r_{i}$ under $E$ in $\mathbb{C}^{2}$ is a complex half-line containing $E(0)$ at its boundary. At the same time it is clear that the field $v_{\mathbb{C}}$ descends to a constant filed $E\left(v_{\mathbb{C}}\right)$ on $\mathbb{C}^{2}$, and so all half-lines of $B(E)$ are contained in the line $L$ through $E(0)$ tangent to $E\left(v_{\mathbb{C}}\right)$.

Since $B(E) \subset L$, the map $E: E^{-1}\left(\mathbb{C}^{2} \backslash L\right) \rightarrow \mathbb{C}^{2} \backslash L$ is a covering map. But the set $E^{-1}\left(\mathbb{C}^{2} \backslash L\right)$ is also a cover of the complement in $C_{K}^{4}$ to all half-planes $\mathbb{R} \times r$ tangent to $v_{\mathbb{C}}$ (including all singular half-planes). We deduce that the last complement is isometric to a product of a punctured 2-cone with $\mathbb{C}$ and the proposition follows. 
Let us sketch an alternative proof of this proposition where instead of studying the enveloping map we work directly with the cone $P^{3}$. Consider the restriction of the field $J(v)$ on $0 \times P^{3}=P^{3}$. The field $J(v)$ is well defined on $P^{3} \backslash 0$, it is preserved by the holonomy of the metric and it is tangent to the singular rays of $P^{3}$. We will show that $P^{3}$ has at most 2 singular rays.

Let $S^{2}$ be the unit sphere centered at the origin of $P^{3}$. Consider the following function on $S^{2}$ :

$$
f: S^{2} \rightarrow[-1,1], \quad f(x)=\cos \left(\angle\left(e_{r}(x), I(v(x))\right) .\right.
$$

We claim that the critical values of this function must be equal to 1 or -1 . Indeed, if $x$ is a nonsingular point and $e_{r}(x)$ is not tangent to $J(v(x))$, then $f$ has nonzero differential at $x$. If $x$ is singular (ie, $x \in r_{i}$ ), then $J(v(x))$ is tangent to $r_{i}$, thus $f(x)= \pm 1$. It follows from Morse theory that $f$ must have exactly two critical points. Thus the number of conical points on $S^{2}$ is at most 2. A further analysis shows that $S^{2}$ is either the unit sphere or a sphere with 2 conical points admitting an isometric $S^{1}$ action preserving the points. This proves the proposition.

Example 3.6 Let $S^{2}$ be a unit sphere and $p$ and $q$ be two points on it. Consider a ramified degree $n$ cover of $S^{2}$ by a sphere $\widetilde{S}^{2}$ with ramifications of order $n$ at $p$ and $q$. Then the pullback metric to $\widetilde{S}^{2}$ has 2 conical points of angles $2 \pi n$. Let $P^{3}$ be a cone over $\widetilde{S}^{2}$ and consider the polyhedral cone $\mathbb{R} \times P^{3}$. The holonomy of the metric on $\mathbb{R} \times P^{3}$ is trivial for all choices of $p$ and $q$, but the cone admits a PK structure only if $p$ and $q$ are opposite points on $S^{2}$. Otherwise, the line $\mathbb{R} \times 0$ forms the singular locus of codimension 3 .

Definition 3.7 Let $C_{K}^{4}$ be a polyhedral Kähler cone. The group $\mathbb{R}^{*}$ is acting on $C_{K}^{4}$ by dilatations (since $C_{K}^{4}$ is a cone). It is clear that this action preserves the holomorphic structure on $C_{K}^{4} \backslash 0$ defined by Proposition 3.5. So the vector field $e_{r}$ generating this action can be complexified and the obtained holomorphic vector field is called Euler vector field and denoted by $e$. The imaginary part of the field $e$ is called the spherical component $e_{s}=J\left(e_{r}\right)$. It is important that $e_{s}$ is acting on $C_{K}^{4}$ by isometries.

Example 3.8 Consider the cone $C_{K}^{4}$ that is the direct product of two 2-cones with conical angles $2 \pi \alpha$ and $2 \pi \beta$. Choose holomorphic coordinates $z$ and $w$ on each $2-$ cone as in the beginning of Section 2.1. Then the Euler field is given by $e=$ $(1 / \alpha) z(\partial / \partial z)+(1 / \beta) w(\partial / \partial w)$. Let us decompose $e$ as above in the radial and spherical components $e=e_{r}+i e_{s}$. If $(\alpha / \beta) \in \mathbb{Q}$, then all orbits of the field $e_{s}$ are closed, and the field $e_{s}$ generates an action of $S^{1}$ on $C_{K}^{4}$. For $(\alpha / \beta)$ irrational, the closure of a generic orbit of $e_{s}$ is a 2-torus. 


\subsection{Linear coordinates on PK cones}

In this subsection we prove Theorem 1.7. In particular, we introduce a holomorphic chart in a neighborhood of every singularity of codimension 4 . So, this also finishes the proof of Theorem 1.5.

Let $C_{K}^{4}$ be a 4-dimensional PK cone. We will consider the isometric action generated by $e_{S}$ on $C_{K}^{4}$ and will distinguish two cases.

(Irrational) There exists at least one nonclosed orbit.

(Rational) All orbits of the action are closed.

Proposition 3.9 If at least one of the orbit of the $e_{S}$ action on $C_{K}^{4}$ is nonclosed then $C_{K}^{4}$ is isometric to the product of two 2-cones.

Consider the group of isometries of $C_{K}^{4}$ preserving its origin. This is a compact Lie group, and the field $e_{s}$ generates its subgroup isomorphic to $\mathbb{R}^{1}$ (because at least one orbit of the action on $S^{3}$ is nonclosed). The closure of this subgroup in the group of isometries is a compact connected Abelian group, ie, a torus of dimension at least two. Thus we have a faithful action of $T^{2}$ on $C_{K}^{4}$ by isometries.

Let us show that the branching locus of the enveloping map $E$ of $C_{K}^{4}$ is contained in the union of two orthogonal lines in $\mathbb{C}^{2}$. Indeed, $T^{2}$ is acting on $C_{K}^{4}$ and this action induces an action of $\mathbb{R}^{2}$ on $\mathbb{C}^{2}$ equivariant with respect to $E$ and fixing the point $E(0)$ in $\mathbb{C}^{2}$. This action factors through the standard action of $T^{2}$ on $\mathbb{C}^{2}$ and it leaves invariant two orthogonal lines $L_{1}$ and $L_{2}$ through $E(0)$. The branching locus of $E$ is a union of lines trough $E(0)$ invariant under $T^{2}$ action. Thus the map

$$
E: E^{-1}\left(\mathbb{C}^{2} \backslash L_{1} \cup L_{2}\right) \rightarrow \mathbb{C}^{2} \backslash L_{1} \cup L_{2}
$$

is a covering map. It follows that $C_{K}^{4}$ is isometric to a product of two 2-cones.

Theorem 1.7 holds for PK cones isometric to the direct product of two 2-cones (see the example above). So the first case of the theorem is proved. To treat the second case we will study the action of $e_{s}$ on the unit sphere $S^{3}$ of $C_{K}^{4}\left(S^{3}\right.$ it the set of points lying at distance 1 from the origin). This action is isometric and we suppose this time that all orbits are closed.

Lemma 3.10 Suppose that all orbits of the action of $e_{s}$ on $S^{3}$ are closed. Then there exists $\alpha>0$ such that all orbits except at most two have period $2 \pi \alpha$, and the exceptional orbits have periods $2 \pi \alpha / p, 2 \pi \alpha / q$, where $p$ and $q$ are coprime numbers. Moreover the action is conjugate to the action $(z, w) \rightarrow\left(e^{i \theta p} z, e^{i \theta q} w\right), \theta \in \mathbb{R} / 2 \pi \mathbb{Z}$ on the unit sphere in $\mathbb{C}^{2},|z|^{2}+|w|^{2}=1$. 
Proof This lemma is standard and follows essentially form the fact that $e_{s}$ is acting on $S^{3}$ by isometries, we will just indicate the proof. It is sufficient to show that the action induces on $S^{3}$ the structure of a Seifert fibration, in particular all orbits apart from a finite number have period $2 \pi \alpha$ and the periods of all exceptional orbits divide $2 \pi \alpha$. Then the lemma follows from the classification of Seifert fibrations on $S^{3}$.

Let $o$ be an orbit of $e_{S}$ on $S^{3}$, denote by $2 \pi \alpha_{1}$ its length. Consider the flow on $S^{3}$ generated by $e_{s}$ in time $2 \pi \alpha_{1}$. It is identical on $o$ and induces a self-map on an invariant slice transversal to $o$. This self-map of the slice is an isometry and it has finite period $n$ (otherwise there exist orbits of $e_{s}$ that are not closed). It follows that the flow generated by $e_{s}$ in time $2 n \pi \alpha_{1}$ induces the identity map on $S^{3}$ thus the period of every orbit divides $2 n \pi \alpha_{1}$. Since every orbit has a neighborhood where all other orbits have period $2 n \pi \alpha_{1}$, the number of exceptional orbits is finite.

Now, we are ready to give the proof of Theorem 1.7 in the second case. Note first that since the field $e_{s}$ defines an $S^{1}$ action on $C_{K}^{4}$, the field $e=e_{r}+i e_{s}$ defines a holomorphic $\mathbb{C}^{*}$ action on $C_{K}^{4} \backslash 0$.

Suppose first that all orbits of the action of $S^{1}$ on $S^{3}$ have the same length, ie, the pair $(p, q)$ from Lemma 3.10 is $(1,1)$. Then the quotient space $\left(C_{K}^{4} \backslash 0\right) / \mathbb{C}^{*}$ is a complex curve homeomorphic to $S^{2}$, hence it is $\mathbb{C} P^{1}$. Thus $C_{K}^{4} \backslash 0$ is isomorphic to a holomorphic $\mathbb{C}^{*}$ fibration over $\mathbb{C} P^{1}$. This fibration can be completed in a unique way to a line bundle by adding the zero section. The completed line bundle has first Chern class -1 , (indeed, the associate $S^{1}$ bundle is homeomorphic to the Hopf fibration of $\left.S^{3}\right)$. We conclude that $C_{K}^{4} \backslash 0$ can be identified with $\mathbb{C}^{2} \backslash 0$ and the Euler field has the form $(1 / \alpha) z(\partial / \partial z)+(1 / \alpha) w(\partial / \partial w)$.

Consider now the case $2 \leq p<q$. Let us reduce it to the case $(p, q)=(1,1)$. According to Lemma 3.10 there are two orbits of the action of $S^{1}$ on $S^{3}$ of lengths $2 \pi(\alpha / p)$ and $2 \pi(\alpha / q)$. Consider the corresponding orbits $O_{p}$ and $O_{q}$ of the action of $\mathbb{C}^{*}$ on $C_{K}^{4}$. It follows from Lemma 3.10 that the triple $\left(C_{K}^{4}, O_{p}, O_{q}\right)$ is homeomorphic to a triple $\left(\mathbb{C}^{2}, \mathbb{C}^{1}, \mathbb{C}^{1}\right)$ composed of a complex plane and two transversal lines. Thus there exists a unique ramified covering of the cone $C_{K}^{4}$ by another polyhedral Kähler cone $\widetilde{C_{K}^{4}}$ of degree $p q$ that has ramifications of orders $p$ over $O_{p}$ and $q$ over $O_{q}$. It is easy to see that constructed cone $\widetilde{C_{K}^{4}}$ has type $(1,1)$ and that there are holomorphic coordinates $(x, y)$ on $\widetilde{C_{K}^{4}}$ such that the Euler field equals $(1 / \alpha) x(\partial / \partial x)+(1 / \alpha) y(\partial / \partial y)$. The holomorphic coordinates on $C_{K}^{4}$ will then be $z=x^{p}, w=y^{q}$ and the Euler field is $e=(p / \alpha) z(\partial / \partial z)+(q / \alpha) w(\partial / \partial w)$.

Definition 3.11 The coordinates $z$ and $w$ constructed above are called linear coordinates of a PK cone. A PK cone is called rational of type $(p, q, \alpha)(p, q \in \mathbb{N})$ if its Euler 
field is equal to $e=(p / \alpha) z(\partial / \partial z)+(q / \alpha) w(\partial / \partial w)$ in the linear coordinates. The number $\alpha$ is called the conical angle of the cone. A cone is called irrational of type $\left(\alpha_{1}, \alpha_{2}\right)$, $\left(\alpha_{1} / \alpha_{2}\right) \in \mathbb{R} / \mathbb{Q}$, if its Euler field is equal to $e=\left(1 / \alpha_{1}\right) z(\partial / \partial z)+\left(1 / \alpha_{2}\right) w(\partial / \partial w)$.

Remark 3.12 For a rational polyhedral Kähler cone of type $(p, q, \alpha)$, all the orbits of the Euler field action are given by the equations $\left(z^{p} / w^{q}\right)=$ const. These curves are flat with respect to the PK metric and all of them (except the curves $z=0, w=0$ ) have the same conical angle at 0 equal to $2 \pi \alpha$.

The proof of the following corollary is contained the second part of the proof of Theorem 1.7.

Corollary 3.13 For a polyhedral Kähler cone $C_{1}$ of type $(p, q, \alpha)$ there exists a unique cone $C_{2}$ of type $(1,1, \alpha)$ with a holomorphic map $f: C_{2} \rightarrow C_{1}$ of degree $p q$ that is a local isometry outside the branching locus.

Finally, we describe all PK cones whose singular locus is a union of two lines in linear coordinates.

Lemma 3.14 Let $C_{K}^{4}$ be a PK cone with linear coordinates $(z, w)$ and such that the singular locus is the union of the lines $z=0$ and $w=0$. Then either $C_{K}^{4}$ is isometric to the product of two 2-cones, or the metric on $C_{K}^{4}$ is the pullback of a constant metric on $\mathbb{C}^{2}$ under the map $\mathbb{C}^{2} \rightarrow \mathbb{C}^{2},(z, w) \rightarrow\left(z^{n}, w^{m}\right)$.

Proof We can suppose that $C_{K}^{4}$ is a rational cone, irrational case is treated by Proposition 3.9. Using Corollary 3.13 we can assume that the cone is of type $(1,1)$, ie, conical angles at the lines $z=0$ and $w=0$ are both equal $2 \pi \alpha$. Now, consider two cases.

(1) $\alpha$ is not integer. Fix a nonsingular point $x$ in $C_{K}^{4}$ and consider the holonomy of the metric based at $x$. This holonomy is generated by two commuting operators $H_{z}$ and $H_{w}$ corresponding to two pathes around lines $z=0$ and $w=0$ (both operators are nontrivial, since $\alpha$ is non integer). Then on $C_{K}^{4} \backslash\{z w=0\}$ we have two holomorphic rank 1 subbundle of $T C_{K}^{4}$ invariant under parallel translation and orthogonal at every point.

Consider the enveloping map $E: C_{K}^{4} \rightarrow \mathbb{C}^{2}$. Invariant subbundles are mapped by $E$ to constant orthogonal subbundles of $T \mathbb{C}^{2}$. It is clear that the ramification locus is composed of two lines through $E(0)$, tangent to one of the constant fields. These lines are orthogonal and so $C_{K}^{4}$ is a direct product of two 2-cones.

(2) $\alpha$ is integer. Then it is clear that the enveloping map from $C_{K}^{4}$ is in fact not multivalued but is a finite degree ramified covering of $\mathbb{C}^{2}$ with ramifications of degree $\alpha$ at the lines $z=0, w=0$. Moreover, the images of both lines are lines in $\mathbb{C}^{2}$ containing $E(0)$, so we are in the second case described by the lemma. 


\section{3 $4_{\mathbb{R}}$ PK cones and $2_{\mathbb{R}}$ spheres with conical points (proofs of Theorems 1.8 and 1.9)}

We start the proof of Theorem 1.8 and associate to every PK cone of type $(1,1, \alpha)$ a metric on a sphere $S^{2}$, of curvature 4 , having conical singularities.

Denote by $S^{3}$ the unit sphere around the origin of the cone and by $S^{2}$ the quotient of $S^{3}$ by the action of $e_{s}$. Locally, outside the singularities, the action of $e_{s}$ on $S^{3}$ is isometric to the action of the Euler field on the standard (nonsingular) unit sphere. Therefore, locally, outside the singularities, the quotient metric on $S^{2}$ is isometric to the quotient of the standard (nonsingular) sphere by $e_{s}$. The last quotient obviously has curvature 4 . The singularities of the cone correspond to the conical points on $S^{2}$.

Lemma 3.15 Let $\Omega$ be a contractible domain on the standard sphere $S^{2}$ of curvature 4 (without conical points). Then, for any positive $l$, there is a unique metric $g$ of curvature 1 on $\Omega \times S^{1}$ with the following properties: All the fibers of the product are geodesics of length $l$; there is an action of $S^{1}$ on $\Omega \times S^{1}$ by isometries; the quotient metric on $\Omega$ coincides with the original one.

Proof Let $\phi: S^{3} \rightarrow S^{2}$ be the standard Hopf fibration. The universal cover of $\phi^{-1}(\Omega)$ is diffeomorphic to $\Omega \times \mathbb{R}$, and $\mathbb{R}$ acts on it by parallel translations. The quotient of $\Omega \times \mathbb{R}$ by the subgroup $l \mathbb{Z}$ of $\mathbb{R}$ induces on $\Omega \times S^{1}$ the metric we are looking for. $\square$

There is a natural connection $\nabla$ on the fibration $\Omega \times \mathbb{R} \rightarrow \Omega$. Its horizontal distribution is given by the planes orthogonal to the fibers. The following lemma is standard and we omit the proof.

Lemma 3.16 The holonomy of the connection $\nabla$ along a closed curve $\gamma \subset \Omega$ is equal to the parallel translation by $2 \operatorname{area}(\gamma)$, where $\operatorname{area}(\gamma)$ is the algebraic area bounded by $\gamma$.

Now, let $S^{2}$ be a sphere with a metric of curvature 4 with conical points. We will associate to it a PK cone of type $(1,1)$.

First, we reconstruct the sphere $S^{3}$ of curvature 1 (with singularities) that fibers over $S^{2}$. Cut $S^{2}$ by geodesic segments with vertices at all the conical points, in order to obtain a contractible polygon $P$. This polygon can be immersed into the standard sphere of curvature 4 by the enveloping map. Consider the fibration over $P$ from Lemma 3.15 with length $l=2 \operatorname{area}(P)$. The holonomy of the fibration along the border of $P$ is trivial (by Lemma 3.16 the circle $S^{1}$ makes one full rotation). This means that the original gluing of $P$, which gives $S^{2}$ with conical points, can be lifted to a 
gluing of $P \times S^{1}$. To construct such a gluing, we choose a horizontal section $s$ of $P \times S^{1}$ over the boundary of $P$ and identify $(x, s(x))$ with $(y, s(y))$ whenever $x$ and $y$ are identified by the gluing of $P$. Since the border circle turns once, we obtain the sphere $S^{3}$.

Now, consider the space $\mathbb{R}_{+} \times S^{3}$ with the metric $(d r)^{2}+r(d s)^{2}$, where $r \in \mathbb{R}_{+}$and $(d s)^{2}$ is the metric constructed on $S^{3}$. This space is a PK cone of type $(1,1)$.

The general case The 2-sphere with conical and marked points associated to the PK cone is given by the metric quotient of the unit sphere in the PK cone by the action of the field $e_{s}$. The marked points correspond to the multiple orbits.

Let $S^{2}$ be a sphere of curvature 4 with conical points two of which, $x$ and $y$, are marked. Let us construct for every $1<p<q$ the corresponding $(p, q)-$ cone. Take first the $(1,1)$-cone $C$ associated to the sphere $S^{2}$ constructed above. Denote by $l_{x}$ and $l_{y}$ the preimages of $x, y \subset C$ under the projection to $S^{2}$. Consider the ramified covering of degree $p q$ over $C$ with the branchings of orders $p$ over $l_{x}$ and of $q$ over $l_{y}$. This is the $(p, q)$-cone we are looking for.

Proof of Theorem 1.9 Consider the case of PK cones of type $(1,1, \alpha)$. Let $S^{2}$ be the quotient sphere associated to the cone. It has $n$ conical points of angles $2 \pi \beta_{1}, \ldots, 2 \pi \beta_{n}$ and its area is given by the Gauss-Bonnet formula:

$$
\operatorname{area}\left(S^{2}\right)=\frac{1}{4}\left(4 \pi+\sum_{i} 2 \pi(\beta-1)\right) .
$$

From Lemma 3.16 it follows $2 \pi \alpha=2 \operatorname{area}\left(S^{2}\right)$. This proves the theorem for $(1,1, \alpha)$ singularities. Singularities of other types are treated in a same way using Corollary 3.13 .

\subsection{PK metrics on singular piecewise linear spaces}

Definition 1.1 can be naturally extended to the following class of PL manifolds with singularities. We call a 4-dimensional topological space with a simplicial decomposition a PL-manifold up to codimension 2 if every 3-simplex is a border of exactly two 4-simplices. A compatible choice of flat metric on the 4-simplices of such a space defines a polyhedral metric on it. Obtained metric has singularities only in codimension 2 and we can repeat Definition 1.1 saying that this metric is a PK metric if its holonomy is contained in $U(2)$ and all singular 2 -faces of conical angles $2 \pi k$ $(k \geq 2)$ have holomorphic directions. A space with such a structure is called a singular PK manifold. 
Most of the theorems of this section about (nonsingular) $4_{\mathbb{R}}$ dimensional PK manifolds can be restated for singular $4_{\mathbb{R}}$ dimensional PK manifolds. In fact, these singular manifolds are complex surfaces with isolated singularities. We will formulate the result but will skip the proof.

Theorem 3.17 For a singular PK manifold of dimension $4_{\mathbb{R}}$ its complex structure defined outside singularities can be extended to the whole singular manifold. Obtained complex space is a complex surface with isolated singularities. In the neighborhood of every isolated singularity there is a natural holomorphic field $e=e_{r}+i e_{s}$ such that the (real) field $e_{r}$ acts by dilatation of the metric and the (real) field $e_{s}$ generates an action of $S^{1}$ by isometries.

\section{Polyhedral Kähler metrics via logarithmic connections}

For every PK surface $S$ the PK metric induces on $T(S \backslash \operatorname{sing})$ a holomorphic, flat, unitary, torsion free connection. This connection extends to a meromorphic connection on $T S$ with first order poles at the singular locus. In this section we will write explicit formulas of PK connections in linear coordinates $z, w$ on 2-dimensional PK cones. We also give a condition for a unitary connection on the tangent bundle of a complex surface that implies that the corresponding metric on $S$ is PK.

\subsection{Definitions and first results}

Let $M$ be a complex manifold and $D$ be a normal crossing divisor. A meromorphic 1 -form $\omega$ on $M$ is called logarithmic with respect to $D$ if it is holomorphic on $M \backslash D$, and in a neighborhood of any point of $D$ it can be represented as

$$
\omega=\sum_{i=1}^{k} f_{i} \frac{d z_{i}}{z_{i}}+\sum_{i=k+1}^{n} f_{i} d z_{i},
$$

where $f_{i}$ are holomorphic functions, $z_{i}$ are local coordinates, and $D$ is given locally by the equation

$$
D=\bigcup_{i=1}^{k}\left\{z_{i}=0\right\} .
$$

The sheaf of logarithmic 1 -forms is denoted by $\Omega^{1}(\log D)$.

Let $E$ be a holomorphic vector bundle over $M$. A meromorphic connection $\nabla$ on $E$ is called logarithmic (with respect to $D$ ) if it can be written in local coordinates as

$$
\nabla=d+A,
$$


where $A$ is a $\Omega^{1}(\log D)$-valued section of $\operatorname{End}(E)$.

For any irreducible component $D_{i}$ of $D$ we denote by $\operatorname{Res}_{D_{i}}(\nabla)$ the residue of $\nabla$ with respect to $D_{i}$, it is a holomorphic section of $\left.\operatorname{End}(E)\right|_{D_{i}}$.

The following proposition is standard, the proof can be found in Section 4 of the article of Malgrange in [1].

Proposition 4.1 Let $\nabla$ be a flat logarithmic connection on $\left(M, E^{k}\right)$ with poles at a normal crossing divisor $D$. Suppose that all eigenvalues of $\operatorname{Res}_{D}(\nabla)$ are contained in ] $-1,0]$.

For a point $x \in D$ chose local coordinates $z_{i}$ such that $D_{i}$ is given by $\bigcup_{i}\left\{z_{i}=0\right\}$. Then there exists a neighborhood $U$ of $x$ and holomorphic sections $s_{1}, \ldots, s_{k}$ of $E^{k}$ giving a trivialisation of $E^{k}$ over $U$ and such that in this trivialisation $\nabla$ is given by

$$
\nabla=d+\sum_{i} B_{i} \frac{d z_{i}}{z_{i}},
$$

where $B_{i}$ are constant matrix-valued functions.

Definition 4.2 For a complex manifold $M$ and a meromorphic connection $\nabla$ on $T M$ its torsion is a meromorphic section of $\Omega^{2}(M) \otimes T M$. It is given by the following formula:

$$
T(u, v)=\nabla_{u}(v)-\nabla_{v}(u)-[u, v],
$$

$u, v \in T M$. A connection with zero torsion is called torsion-free.

Now, we will restrict our attention to connections on the tangent bundles of surfaces. Let $S$ be a surface with a weighted arrangement of curves $\left(\Gamma_{j}, \beta_{j}\right)$ and let $x_{1}, \ldots, x_{k}$ be the points of the arrangement of multiplicity at least 3 .

Definition 4.3 We say that a meromorphic connection $\nabla$ on $T S$ is partially adapted to $\left(\Gamma_{j}, \beta_{j}\right)$ if $\nabla$ is logarithmic on $S \backslash\left\{x_{1}, \ldots, x_{k}\right\}$, $\operatorname{Res}_{\Gamma_{j}} \nabla$ has eigenvalues $\left(\beta_{j}-1,0\right)$ at $\Gamma_{j}$, and $T \Gamma_{j} \cong \operatorname{ker}\left(\operatorname{Res}_{\Gamma_{j}} \nabla\right)$.

Lemma 4.4 On every PK surface $S$ the PK connection is partially adapted to the weighted arrangement of $S$.

Proof The statement of the lemma clearly holds at smooth points of the singular locus of $S$, because they can be embedded isometrically in the product of $\mathbb{C}$ with a 2-cone. At the same time the connection on the $2-$ cone is logarithmic and has residue $\beta-1$ where $2 \pi \beta$ is the cone angle. 
From the description of double points of the singular locus (Lemma 3.14) it follows that the connection is also logarithmic at these points. Indeed, the connection on the direct product of two 2-cones is logarithmic, and for the second type of cones that are branched covers of $\mathbb{C}^{2}$ one can change the metric without changing the connection to make these cones also direct products.

Lemma 4.5 Let $S$ be a complex surface with a weighted arrangement $\left(\Gamma_{j}, \beta_{j}\right)$, $\beta_{j} \neq 1$. Suppose that $\nabla$ is partially adapted, then its torsion is holomorphic.

Proof By Hartog's theorem it is sufficient to show that the torsion of $\nabla$ is holomorphic outside of the multiple points of the arrangement $\Gamma_{j}$. So it is sufficient to consider the case of a connection on $\mathbb{C}^{2}$ with a pole at the line $z=0$. Chose locally the second coordinate $w$ in such a way that the residue of $\nabla$ is given by the formula

$$
\operatorname{Res}_{z=0} \nabla=\left(\begin{array}{cc}
\beta-1 & 0 \\
0 & 0
\end{array}\right) \text {. }
$$

In this case the connection $\nabla$ can be written as

$$
\nabla=d+\frac{d z}{z} \operatorname{Res}_{z=0} \nabla+A,
$$

where $A$ is holomorphic. This proves the lemma.

Corollary 4.6 Suppose that $\Gamma\left(\Omega_{S}^{1}\right)=0$, ie, there is no nontrivial holomorphic 1forms on $S$. Then the torsion of $\nabla$ is identically zero.

Proof The torsion of a meromorphic connection on the tangent bundle to a complex manifold $X$ is a section of $\Omega_{X}^{2} \otimes T X$. In the case when $X$ is a two-dimensional complex surface $S$ the bundle $\Omega_{S}^{2} \otimes T S$ is two-dimensional and isomorphic to the bundle of holomorphic 1-forms $\Omega_{S}^{1}$. Thus on $S$ any holomorphic section of $\Omega_{S}^{2} \otimes T S$ is identically zero.

\subsection{Formulas for connections on $(1,1)$ cones}

In the following proposition we describe the connection of a PK metric in a neighborhood of a singular point of type $(1,1)$.

Proposition 4.7 Consider a PK cone of type $(1,1, \alpha)$ with linear coordinates $z, w$. For $i=1, \ldots, n$ let $l_{1}=0, \ldots, l_{n}=0$ be the equations of the singular lines of the cone. Let $2 \pi \beta_{i}$ be the conical angle at $l_{i}=0$. Then the following holds: 
(1) The residue $\operatorname{Res}_{l_{i}} \nabla$ of $\nabla$ at $l_{i}$ is given by a constant matrix-valued function $A_{i}$ and the connection $\nabla$ is given by the following formula:

$$
\nabla=d+A=d+\sum_{i=1}^{n} A_{i} \frac{d l_{i}}{l_{i}} .
$$

(2) The residues $A_{i}$ satisfy the following equations:
(a) $\sum_{i=1}^{n} A_{i}=(\alpha-1) \mathrm{Id}$
(b) $\operatorname{tr}\left(A_{i}\right)=\beta_{i}-1$;
(c) $\left\{l_{i}=0\right\}=\operatorname{ker}\left(A_{i}\right)$.

Proof of Proposition 4.7(1) We prove first that the residue of $\nabla$ is constant at any $l_{i}$. Indeed, the action of $\mathbb{C}^{*}$ on the cone changes the PK metric by a scalar factor, thus this action preserves the connection $\nabla$. For any $c \in \mathbb{C}^{*}$ we have

$$
A(c z, c w)=A(z, w),
$$

ie, the residue $\operatorname{Res}_{l_{i}}(\nabla)$ is constant on $l_{i}$.

Consider now the following connection $\nabla^{\prime}$ on $\mathbb{C}^{2}$ :

$$
\nabla^{\prime}=d+\sum_{i} \operatorname{Res}_{l_{i}}(\nabla) \cdot \frac{d l_{i}}{l_{i}} .
$$

We claim that $\nabla^{\prime}=\nabla$. Indeed, the matrix-valued 1 -form $\nabla^{\prime}-\nabla$ has no poles at the lines $l_{i}$ thus it is holomorphic on $\mathbb{C}^{2}$. Moreover this 1 -form is preserved by the $\mathbb{C}^{*}$ action, ie, it is identically zero.

Next lemma is essential for the proof of Proposition 4.7(2).

Lemma 4.8 For the Euler field $e$ of the PK cone we have

$$
\nabla_{e} e=e .
$$

Proof It is sufficient to check this identity for the flat $\mathbb{C}^{2}$.

Proof of Proposition 4.7(2) Let us prove (4-3)(a). The Euler field $e$ on the PK cone is given by

$$
e=\frac{1}{\alpha}\left(z \frac{\partial}{\partial z}+w \frac{\partial}{\partial w}\right)
$$

We have

$$
\nabla_{e} e=d e(e)+\sum_{i=1}^{n} A_{i}(e) \frac{d l_{i}}{l_{i}}(e)=\frac{1}{\alpha} e+\sum_{i=1}^{n} \frac{1}{\alpha} A_{i}(e)=e
$$


This means that $\sum_{i=1}^{n} A_{i}(e)=(\alpha-1) e$, ie, $A=(\alpha-1)$ Id.

Statements (4-3)(b)-(c) are proven in Lemma 4.4.

Remark 4.9 For $i=1, \ldots, n$ let $l_{i}$ be lines in $\mathbb{C}^{2}$ containing the origin and let $\beta_{i}$ be complex numbers. Then the space of matrixes $A_{i}$ satisfying (4-3) has dimension $(n-3)$. Next formula gives the unique connection for $n=3$ with poles at the lines $z=0, w=0, z+w=0$ :

$$
\nabla=d+\left(\begin{array}{cc}
\left(\beta_{1}-1\right) \frac{d z}{z}+\frac{\beta_{2}+\beta_{3}-\beta_{1}-1}{2} \frac{d z+d w}{z+w} & \frac{\beta_{2}+\beta_{3}-\beta_{1}-1}{2}\left(\frac{d z+d w}{z+w}-\frac{d w}{w}\right) \\
\frac{\beta_{1}+\beta_{3}-\beta_{2}-1}{2}\left(-\frac{d z}{z}+\frac{d z+d w}{z+w}\right) & \frac{\beta_{1}+\beta_{3}-\beta_{2}-1}{2} \frac{d z+d w}{z+w}+\left(\beta_{2}-1\right) \frac{d w}{w}
\end{array}\right)
$$

Proposition 4.10 Any connection $\nabla$ on $\mathbb{C}^{2}$ given by formula (4-2) with the matrices $A_{i}$ satisfying (4-3) is flat, torsion-free and thus it defines a singular affine structure on $\mathbb{C}^{2}$.

Proof Since $d A=0$ the curvature of $\nabla$ is given by

$$
d A+A \wedge A=A \wedge A
$$

We need to prove that $A \wedge A=0$. Writing

$$
A_{i}=\left(\begin{array}{cc}
a_{i} & b_{i} \\
c_{i} & d_{i}
\end{array}\right)
$$

then the equation $A \wedge A=0$ is equivalent to the following system:

$$
\begin{cases}\sum_{i} c_{i} \frac{d l_{i}}{l_{i}} \wedge \sum_{i} b_{i} \frac{d l_{i}}{l_{i}} & =0 \\ \sum_{i} b_{i} \frac{d l_{i}}{l_{i}} \wedge \sum_{i}\left(a_{i}-d_{i}\right) \frac{d l_{i}}{l_{i}} & =0 \\ \sum_{i} c_{i} \frac{d l_{i}}{l_{i}} \wedge \sum_{i}\left(a_{i}-d_{i}\right) \frac{d l_{i}}{l_{i}} & =0\end{cases}
$$

For the first equation we have

$$
\sum_{i} c_{i} \frac{d l_{i}}{l_{i}} \wedge \sum_{i} b_{i} \frac{d l_{i}}{l_{i}}=d \log \left(\prod_{i} l_{i}^{c_{i}}\right) \wedge d \log \left(\prod_{i} l_{i}^{b_{i}}\right) .
$$

Function $f_{1}=\log \left(\prod_{i} l_{i}^{c_{i}}\right)$ and $f_{2}=\log \left(\prod_{i} l_{i}^{b_{i}}\right)$ are homogeneous of degree 0 on $\mathbb{C}^{2}$ (since $\sum c_{i}=\sum b_{i}=0$ by (4-3)(a)). It follows that $d f_{1} \wedge d f_{2}=0$. The next two equations are completely analogous. 
Now we show that $A$ is torsion free. We have

$$
\begin{aligned}
T\left(\frac{\partial}{\partial z}, \frac{\partial}{\partial w}\right) & =\sum_{i=1}^{n} \frac{d l_{i}}{l_{i}}\left(\frac{\partial}{\partial z}\right) A_{i}\left(\frac{\partial}{\partial w}\right)-\frac{d l_{i}}{l_{i}}\left(\frac{\partial}{\partial w}\right) A_{i}\left(\frac{\partial}{\partial z}\right) \\
& =\sum_{i=1}^{n} \frac{1}{l_{i}} A_{i}\left(\frac{\partial l_{i}}{\partial z} \frac{\partial}{\partial w}-\frac{\partial l_{i}}{\partial w} \frac{\partial}{\partial z}\right)=0 .
\end{aligned}
$$

The last equality holds by (4-3)(c).

\section{Connection on the blow-up}

Lemma 4.11 Let $\nabla$ be a connection on $\mathbb{C}^{2}$ given by formula (4-2) with the matrices $A_{i}$ satisfying (4-3). Consider the blow-up of $\mathbb{C}^{2}$ at $(0,0)$, take the pullback of the tangent bundle of $\mathbb{C}^{2}$ to the blow-up, and consider on it the pullback of $\nabla$. The obtained connection is logarithmic and its residue at the exceptional curve is $(\alpha-1) \mathrm{Id}$.

Proof Let us introduce coordinates $u, v$ on the blow-up $u=(z / w), v=w$. The exceptional line is given by $v=0$. Let $l_{i}=s_{i} z+t_{i} w=s_{i} u v+t_{i} v$. Then the pullback connection is given by

$$
\begin{aligned}
\nabla & =d+\sum_{i=1}^{n} A_{i} d\left(\log l_{i}\right)=d+\sum_{i=1}^{n} A_{i}\left(d(\log v)+d\left(\log \left(s_{i} u+t_{i}\right)\right)\right) \\
& =d+(\alpha-1) \mathrm{Id} \cdot d(\log v)+\sum_{i=1}^{n} A_{i} \cdot d\left(\log \left(s_{i} u+t_{i}\right)\right) .
\end{aligned}
$$

Here we use Equation (4-3)(a). This proves the lemma.

\subsection{Unitary flat logarithmic torsion free connection $\mapsto$ PK metric}

In this subsection we consider only arrangements $\left(S, \Gamma_{j}\right)$ that satisfy the property that $\Gamma_{j}$ are smooth and transversal. We give a sufficient criterion for such an arrangement to be the singular locus of a PK metric in terms of an adapted connection. Singularities of such an arrangement are normal crossings or singularities of type $(1,1)$.

Definition 4.12 Let $\nabla$ be a connection partially adapted to $\left(S, \Gamma_{j}, \beta_{j}\right)$. Suppose that $\Gamma_{j}$ are smooth and intersect transversally. Consider the blow up $\pi: \widetilde{S} \rightarrow S$ at all points $x_{i}$ of multiplicity at least $3 . \nabla$ is adapted to $\left(\Gamma_{j}, \beta_{j}\right)$ if the pullback connection $\pi^{*} \nabla$ on $\pi^{*} T S$ is logarithmic on $\widetilde{S}$ and its residue at the exceptional curve over $x_{i}$ equals $\sum_{j} d_{i j}\left(\beta_{j}-1\right) \mathrm{Id}$. 
Theorem 4.13 Let $\left(S, \Gamma_{j}, \beta_{j}, x_{i}\right)$ be a weighted arrangements of curves and $\nabla$ be an adapted flat, unitary, torsion free connection on TS. Suppose that $0<\beta_{j}<1$ and for every $x_{i}, \sum_{j} d_{i j}\left(\beta_{j}-1\right)>-2$. Then the unitary metric on $T S$ corresponding to $\nabla$ is a $\mathrm{PK}$ metric.

Proof Note first that since $\nabla$ is unitary, flat, and torsion-free, the metric $g$ corresponding to $\nabla$ is flat on the complement to the curves $\Gamma_{j}$. So to prove that $g$ extends to a PK metric on $S$ it will be sufficient to show the following 3 properties of $g$.

(a) For any smooth point of $\Gamma$ there is a neighborhood $U$ with $U \backslash \Gamma$ isometric to the direct product of a flat punctured 2-cone and a flat disc.

(b) For any double point of $\Gamma$ there is a neighborhood $U$ with $U \backslash \Gamma$ isometric to the direct product of two flat punctured 2-cones.

(c) For any point of $\Gamma$ of multiplicity greater than 2 a neighborhood of the point is isometric to a $2 \mathbb{C}$-dimensional PK cone.

The proofs of (a) and (b) are similar so we will prove only (b) and (c).

Proof of (b) Introduce coordinates $z, w$ in a neighborhood of the double point such that $\Gamma$ is locally given by $z w=0$. According to Proposition 4.1 there exist two sections $s_{1}$ and $s_{2}$ such that the connection $\nabla$ is given by

$$
\nabla=d+B_{1} \frac{d z}{z}+B_{2} \frac{d w}{w},
$$

where $B_{1}$ and $B_{2}$ are constant and commuting. Making an additional linear change in $s_{1}, s_{2}$ we can suppose that $B_{1}\left(s_{1}\right)=0$ and $B_{2}\left(s_{2}\right)=0$. It is clear then the subbundles of the tangent bundle generated by $s_{1}$ and $s_{2}$ are invariant under $\nabla$ and moreover the vector field $s_{1}$ is tangent to the line $w=0$ and $s_{2}$ is tangent to the line $z=0$. Since $\nabla$ is torsion free the integral curves of $s_{1}$ and $s_{2}$ are flat cones. So we can deduce that locally the neighborhood of $(0,0)$ is the direct product of two 2 -cones. $\square$

Proof of (c) Let 0 be a point of $\Gamma$ of multiplicity greater than 2. From (a) it follows that the metric $g$ extends continuously to any punctured curve $\Gamma_{j} \backslash 0$, thus we obtain a polyhedral Kähler metric on $U \backslash 0$. It is necessary to show further that $g$ extends to 0 and the resulting metric on $U$ is a polyhedral Kähler metric. To show this it is sufficient to construct the action of $\mathbb{C}^{*}$ on $U \backslash 0$ by dilatations, ie, $\mathbb{R}^{*}$ must act by dilatations of the metric and $S^{1}$ must act by isometries. 
Construction of the $\mathbb{C}^{*}$ action on $U \backslash 0$ Consider the holonomy representation of the group $\pi_{1}(U \backslash \Gamma, x)$ in the group $U(2, \mathbb{C}) \ltimes \mathbb{C}^{2}$ of unitary affine transformations of $\mathbb{C}^{2}$ :

$$
\mathrm{Hol}_{\nabla}: \pi_{1}(U \backslash \Gamma, x) \rightarrow U(2, \mathbb{C}) \ltimes \mathbb{C}^{2} .
$$

The linear part of this representation is denoted by hol $\nabla$. Denote by $c$ the generator of the center of $\pi_{1}(U \backslash \Gamma, x)$ corresponding to an anti-clockwise path around 0 in a complex line through 0. From condition (2) of Definition 4.12 it follows that

$$
\operatorname{hol}_{\nabla}(c)=\exp \left(2 \pi i \sum_{j}\left(\beta_{j}-1\right)\right) \mathrm{Id} \text {. }
$$

We see that the affine transformation $\operatorname{Hol}_{\nabla}(c)$ is a complex rotation of $\mathbb{C}^{2}$ around some point $y$ on angle $2 \pi \sum_{j}\left(\beta_{j}-1\right)$.

Since any element $h$ of $\pi_{1}(U \backslash \Gamma, x)$ commutes with $c$, the affine transformation $\operatorname{Hol}_{\nabla}(h)$ fixes the point $y$. It follows that the representation $\operatorname{Hol}_{\nabla}(c) \pi_{1}(U \backslash \Gamma, x)$ commutes with the affine action of $\mathbb{C}^{*}$ on $\mathbb{C}^{2}$, given by complex dilatations that fix $y$. We deduce that the action of $\mathbb{C}^{*}$ can be pulled back to the action of $\mathbb{C}^{*}$ on $U \backslash 0$.

Remark 4.14 The condition $\beta_{j}<1$ in this theorem can be replaced by the condition $\beta_{j} \notin \mathbb{Z}_{+}$but we don't prove this here.

\section{Topological relations on the singular locus}

In this section we prove Theorem 1.11. We use a formula of Ohtsuki [15].

Theorem [15] Let $S$ be a compact surface and $E$ be a holomorphic vector bundle on it. Let $D=\bigcup_{j} D_{j}$ be a normal crossings divisor on $S$ and $\nabla$ a logarithmic connection on $E$ with poles at $D$. Denote by $y_{k}$ the double points of $D$ and by $D_{k_{1}}$ and $D_{k_{2}}$ the irreducible components of $D$ containing $y_{k}$. We also use the notation $\mathrm{R}_{j}=\operatorname{Res}_{D_{j}}(\nabla)$. The following identities hold:

$$
\begin{aligned}
c_{1}(E)= & -\sum_{j} \operatorname{Tr}\left(\mathrm{R}_{j}\right) D_{j}, \\
c_{2}(E)= & \sum_{k}\left(\operatorname{Det}\left(\mathrm{R}_{k 1}+\mathrm{R}_{k 2}\right)-\operatorname{Det}\left(\mathrm{R}_{k 1}\right)-\operatorname{Det}\left(\mathrm{R}_{k 2}\right)\right)\left(y_{k}\right) \\
& \quad+\sum_{j} \operatorname{Det}\left(\mathrm{R}_{j}\right) D_{j} \cdot D_{j} .
\end{aligned}
$$

Note that the value of the first summand is defined only at $y_{k}$ but the function $\operatorname{Det}\left(\mathrm{R}_{j}\right)$ is defined on the whole divisor $D_{j}$ and is constant on it. 


\subsection{Proof of Theorem 1.11, Equations (1-1) and (1-2)}

Lemma 5.1 For any $j \neq k$ we have

$$
\Gamma_{j} \cdot \Gamma_{k}=B_{j k}+\sum_{i}\left(p_{i} q_{i}\right)\left(d_{i j} d_{i k}\right) .
$$

Proof This formula expresses the intersection index of $\Gamma_{j}$ and $\Gamma_{k}$ as a sum of local multiplicities of their intersections. By definition $B_{j k}$ is the number of transversal intersections of $\Gamma_{j}$ and $\Gamma_{k}$. The local multiplicity of the intersection of $\Gamma_{j}$ and $\Gamma_{k}$ at $x_{i}$ equals $\left(p_{i} q_{i}\right)\left(d_{i j} d_{i k}\right)$. Indeed, the local multiplicity of the intersection of the curves $c z^{q}=w^{p}$ and $z^{q}=w^{p}$ at 0 equals $p q$ if $1 \neq c \neq 0$; the local multiplicities of intersection of $z^{q}=w^{p}$ with lines $z=0$ and $w=0$ are equal to $p$ and $q$ correspondingly. Now everything follows from the definition of $d_{i j}$ and $d_{i k}$.

\section{Proof of Theorem 1.11, Equation (1-1)}

The Gauss-Bonnet theorem for flat surfaces with conical singularities implies

$$
2 g\left(\Gamma_{j}\right)-2=\sum_{k \neq j} B_{j k}\left(\beta_{k}-1\right)+\sum_{i}\left(d_{i j} \alpha_{i}-\tilde{d}_{i j}\right) .
$$

This formula expresses the Euler characteristics of $\Gamma_{j}$ as the sum of the defects of the conical points of $\Gamma_{j}$. The first sum contains the contribution of the normal crossings of $\Gamma_{j}$ and the second sum contains the contribution of the singularities $x_{i}$.

Now, using Theorem 1.9, we obtain the following expression for the right term of the previous equation:

$$
\sum_{k \neq j} B_{j k}\left(\beta_{k}-1\right)+\sum_{i}\left(d_{i j} \frac{p_{i} q_{i}}{2} \sum_{k} d_{i k}\left(\beta_{k}-1\right)+d_{i j} \frac{p_{i}+q_{i}}{2}-\tilde{d}_{i j}\right) .
$$

Using Lemma 5.1, this equals

$$
\begin{aligned}
\sum_{k \neq j} B_{j k} \frac{\beta_{k}-1}{2}+\sum_{k} \Gamma_{j} \cdot \Gamma_{k} & \frac{\beta_{k}-1}{2}-\Gamma_{j} \cdot \Gamma_{j} \frac{\beta_{j}-1}{2}+ \\
& +\sum_{i} \frac{p_{i} q_{i}}{2}\left(d_{i j}\right)^{2}\left(\beta_{j}-1\right)+\sum_{i}\left(d_{i j} \frac{p_{i}+q_{i}}{2}-\tilde{d}_{i j}\right) .
\end{aligned}
$$

Using relation (1-2), this becomes

$$
\sum_{k} B_{j k} \frac{\beta_{k}-1}{2}+\frac{K_{S} \cdot \Gamma_{j}}{2}+\sum_{i}\left(d_{i j} \frac{p_{i}+q_{i}}{2}-\tilde{d}_{i j}\right) .
$$

This proves Theorem 1.11, Equation (1-1). 
Proof of Theorem 1.11, Equation (1-2) Let $S$ be a PK surface and $\left(\Gamma_{j}, \beta_{j}, x_{i}\right)$ be the corresponding weighted arrangement. By Lemma 4.4 the corresponding connection $\nabla$ on $T S$ is logarithmic on $S \backslash \bigcup_{i} x_{i}$. Furthermore $\nabla$ induces a flat connection $\nabla_{K}$ on $K_{S}$ logarithmic on $S \backslash \bigcup_{i} x_{i}$, and with residue $1-\beta_{j}$ at $\Gamma_{j}$. Consider a resolution of singularities of $\Gamma$ on $S, p: S^{\prime} \rightarrow S$ such that $p^{*}(\Gamma)$ is a curve with normal crossing singularities. It is not hard to show that the pullback connection $p^{*} \nabla_{K}$ on the pullback line bundle $p^{*} K_{S}$ is logarithmic on the whole $S^{\prime}$. To prove the statement of the theorem it is sufficient to show that

$$
p^{*}\left[K_{s}\right]-\sum_{j}\left(\beta_{j}-1\right) p^{*}\left[\Gamma_{j}\right]=0 \in H_{2}\left(S^{\prime}\right)
$$

Now notice that by (5-1) this homology class is expressed as a combination of homology classes of exceptional divisors (since the residue of $p^{*} \nabla_{K}$ at $p^{*} \Gamma_{j}$ is $\left(1-\beta_{j}\right)$ ). But since at the same time this class is a pullback from $S$ it must vanish.

\subsection{Proof of Theorem 1.11, Equation (1-3)}

Consider a PK surface $S$ with the weighted arrangement $\left(\Gamma_{j}, \beta_{j} ; x_{i}, \alpha_{i}\right)$ such that all multiple points of the arrangement are either normal crossings or singularities of type $(1,1)$. Let $S_{b}$ be the blow-up of $S$ at the points $x_{i}$ and let $\pi: S_{b} \rightarrow S$ be the blow-down. Denote by $P_{i}$ the exceptional curve over $x_{i}$ and denote by $\widetilde{\Gamma}_{j}$ the proper transform of $\Gamma_{j}$. Consider the pullback $\widetilde{\nabla}$ of the PK connection $\nabla$ to $\pi^{*}(T S)$. By Lemma $4.11 \widetilde{\nabla}$ is logarithmic with poles at the divisor $\bigcup_{i} P_{i} \bigcup_{j} \widetilde{\Gamma}_{j}$ (further we call this divisor by $D)$, and the residue of $\widetilde{\nabla}$ at $P_{i}$ is equal to $\left(\alpha_{i}-1\right)$ Id.

Proof of Theorem 1.11, Equation (1-3) According to (5-2) the number $c_{2}\left(\pi^{*} T S\right)$ can be expressed as the sum of the contributions of the irreducible components of $D$ and the sum over their pairwise intersections. The first sum is the following:

$$
\sum_{i} \operatorname{Det}\left(\operatorname{Res}_{P_{i}} \widetilde{\nabla}\right) P_{i} \cdot P_{i}+\sum_{j} \operatorname{Det}\left(\operatorname{Res} \widetilde{\Gamma}_{j} \widetilde{\nabla}\right) \widetilde{\Gamma}_{j} \cdot \widetilde{\Gamma}_{j}=-\sum_{i}\left(\alpha_{i}-1\right)^{2}
$$

Here we use $\operatorname{Det}\left(\operatorname{Res}_{\widetilde{\Gamma}_{j}} \widetilde{\nabla}\right)=0$.

The sum of the contributions of the double points on $P_{i}$ is the following:

$$
\left(\alpha_{i}-1\right) \sum_{j} d_{i j}\left(\beta_{j}-1\right)=2\left(\alpha_{i}-1\right)^{2}
$$


Any intersection of $\widetilde{\Gamma}_{j}$ with $\widetilde{\Gamma}_{k}$ contributes $\left(\beta_{j}-1\right)\left(\beta_{k}-1\right)$ thus the sum over all intersections of curves $\widetilde{\Gamma}_{j}$ is given by

$$
\sum_{j>k} B_{j k}\left(\beta_{j}-1\right)\left(\beta_{k}-1\right) .
$$

Finally, taking the sum of all contribution we obtain

$$
c_{2}(T S)=c_{2}\left(\pi^{*}(T S)\right)=\sum_{i}\left(\alpha_{i}-1\right)^{2}+\sum_{j>k} B_{j k}\left(\beta_{j}-1\right)\left(\beta_{k}-1\right) .
$$

\subsection{Line arrangements in $\mathbb{C P}^{2}$}

In this subsection we consider weighted arrangements of lines on $\mathbb{C} P^{2}$ that satisfy Equations (1-1)-(1-3). Let $\left(L_{1}, \beta_{1} ; \ldots ; L_{n}, \beta_{n}\right)$ be such an arrangement. Any singularity of this arrangement is either a normal crossing or a $(1,1)$-type singularity. So Equations (1-1) and (1-2) simplify and take the form:

$$
\sum_{k} B_{j k}\left(\beta_{k}-1\right)=-1, \quad \sum_{k}\left(\beta_{k}-1\right)=-3 .
$$

Here by definition the number $B_{i j}(i \neq j)$ is equal to 1 if the point of the intersection of $L_{i}$ and $L_{j}$ is a double point (ie, other lines of the arrangement don't contain it) and $B_{i j}=0$ otherwise. The number $\left(B_{j j}+1\right)$ is equal to the number of points of multiplicity at least 3 on the line $L_{j}$.

Symmetric case Consider the most symmetric case when all angles $\beta_{j}$ are equal. Then we have

$$
\beta_{j}-1=-\frac{3}{n}, \quad \sum_{k} B_{j k}=\frac{n}{3} .
$$

The number $\sum_{k} B_{j k}+1$ is equal to the number of all intersections of $L_{j}$ with other lines. Thus we obtain the following condition:

The arrangement contains $3 m$ lines and any line intersects the other lines exactly at $m+1$ points $(m=n / 3)$.

One can show that such arrangements satisfy as well Equation (1-3). These arrangements were considered first by Hirzebruch in [7] and we recall several examples (all arrangements apart from the first one are PK).

(1) (3 lines) A generic configuration of 3 lines on $\mathbb{C} P^{2}$.

(2) (6 lines) The configuration of 6 lines $x-y=0, x \pm z=0, y \pm z=0 z=0$. 
(3) $(3(m+1)$ lines, $m>1)$ Consider the ramified covering of $\mathbb{C} P^{2}$ by itself given by $(x: y: z) \rightarrow\left(x^{m}: y^{m}: z^{m}\right)$. The preimage of the configuration of 6 lines is an arrangement of $3(m+1)$ lines and any line has $m+2$ intersections with the other lines.

(4) (Hesse arrangement) Consider a nonsingular cubic in $\mathbb{C} P^{2}$. It has 9 points of inflections. There exist 12 lines in $\mathbb{C} P^{2}$ that intersect the cubic exactly at the points of inflections.

Criterion There exists one geometric condition that often permits to decide quickly that a given line arrangement is not PK.

Proposition 5.2 Every PK arrangement $\left(L_{j}, \beta_{j}\right)$ in $\mathbb{C} P^{2}$ satisfy the following criterium. For every $L_{j}$ there exists a point in $\mathbb{C} P^{2}$ that belongs to all lines $L_{k}$ such that $L_{k} \cap L_{j}$ is a double point of the arrangement.

Proof Consider the subbundle of $\left.T \mathbb{C} P^{2}\right|_{L_{j}}$ of directions orthogonal to $L_{j}$ with respect to PK metric (these directions are eigenvectors of the residue map $\operatorname{Res}_{L_{j}} \nabla$ ). This subbundle is holomorphic outside of the multiple points of the arrangement and it extends holomorphically on the whole line $L_{j}$. Indeed, at double points the PK metric is a direct product of two 1-cones, and at every point of multiplicity more than 2 the eigenvectors of $\operatorname{Res}_{L_{j}} \nabla$ are constant in the local linear coordinates (Proposition 4.7). The defined subbundle of $\left.T \mathbb{C} P^{2}\right|_{L_{j}}$ is transversal to $L_{j}$ and so it has degree 1 . It follows that there exists a point $y_{j}$ in $\mathbb{C} P^{2}$ such that this subbundle of $\left.T \mathbb{C} P^{2}\right|_{L_{j}}$ is given by directions tangent to the lines through $y_{j}$. This proves the proposition.

\subsection{Limit PK arrangements with a cusp}

Definition 5.3 An arrangement of lines on $\mathbb{R} P^{2}$ is called simplicial if it subdivides $\mathbb{R} P^{2}$ in triangles.

Simplicial arrangements often occur as solutions to some extreme (combinatorial) problems (see Grünbaum [6]) and so it is not very surprising that some of simplicial arrangements are PK (PK arrangements give an extremum for the Bogomolov-Gieseker Inequality (1-5) in Theorem 1.12). For the moment 3 infinite families of simplicial plane arrangements and 91 sporadic examples are know; 90 sporadic examples are listed by Grünbaum in [5] and one additional in [6]. It will be interesting to find out what sporadic arrangements are PK, the criterium from Proposition 5.2 rules out some of them. 
For three infinite series of arrangements from [5] there exists a system of weights such that all equalities of Theorem 1.11 and Theorem 1.12 are satisfied. The first series is a union of a pencil of $n$ lines and a line that does not belong to the pencil. For this series the weight of the line should be 0 and the weights of the lines from the pencil can be arbitrary (but we impose of course $\sum_{j}\left(\beta_{j}-1\right)=-3$ ). Second series is called $R_{2 k}$ and consists of the lines formed by extending the sides of a regular $k$-gon together with an additional $k$ lines formed be the axes of symmetry of the $k$-gon. We associate to the axes weight $(k-1) / k$ and to the sides weight $(2 k-1) /(2 k)$ (the choice of wights is unique for $k>3$ ). The third series $R_{4 k+1}$ is the union of $R_{4 k}$ with the line at infinity of weight 1 . We see that for the point of the highest multiplicity of these arrangements the inequality $\sum_{j} d_{i j}\left(\beta_{j}-1\right)>-2$ does not hold strictly but instead of this the equality holds. So these arrangements are not PK. We conjecture instead that there is a different geometric structure related to these arrangements.

Definition 5.4 A weighted arrangement $\left(L_{j}, \beta_{j}\right)$ in $\mathbb{C} P^{2}$ is called a limit PK arrangement with a cusp if it satisfies all conditions of Theorem 1.12 apart from one inequality. We impose that there is a multiple point $x$ of the arrangement called the cusp such that $\sum_{j \mid x \in L_{j}}\left(\beta_{j}-1\right)=-2$.

Arrangements $R_{2 k}$ satisfy this definition, $R_{4 k+1}$ and a pencil of lines plus a line formally don't satisfy (because the first arrangement has a line of weight 1 and the second has a line of weight 0 ). But the following should hold for all 3 series.

Conjecture 5.5 For every limit PK arrangement with a cusp there exists a flat torsionfree connection on $\mathbb{C} P^{2}$ with holonomy in the upper triangular subgroup of $\operatorname{SL}(2, \mathbb{C})$ and with the poles of residues $\left(0, \beta_{j}-1\right)$ at the lines $L_{j}$. This connection should preserve the sub-line bundle of $T \mathbb{C} P^{2}$ tangent to the pencil of lines through the cusp of the arrangement.

For a pencil of lines plus a line the connection on $\mathbb{C} P^{2}$ should be given by a formula from Proposition 4.10. Here $\mathbb{C} P^{2}$ is the completion of $\mathbb{C}^{2}$, the line at infinity belongs to the arrangement and has weight $\beta=0$.

If Conjecture 5.5 holds it should be possible to deduce that limit PK arrangements with a cusp satisfy the following restrictive properties.

Conjectural properties For every multiple point $y$ of the arrangement the line $[x, y]$ belongs to the arrangement (here $x$ is the cusp). If $\operatorname{mult}(y) \geq 3$ then sum of the defects of the lines $L_{j}$ that contain $y$ but don't contain $x$ equals the defect of the line $[x, y]$. 
It will be very interesting to classify all weighted arrangements that satisfy the two conjectural properties (they hold for $R_{2 k}$ and $R_{4 k+1}$ ). This will help to classify nonrigid PK arrangements for which the admissible collection of weights $\beta_{j}$ have moduli. Of course the weights $\beta_{j}$ belong to a certain open polyhedron and the weights corresponding to limit PK arrangements can appear on the boundary of the polyhedron.

\section{Parabolic bundles and Kobayashi-Hitchin correspondence}

The goal of this section is to recall the notion of parabolic bundles and to formulate in a handy way several results from Mochizuki [13] that we use in the proof of Theorem 1.12. In particular we formulate the parabolic version of Kobayashi-Hitchin correspondence from [13]. A systematic and thorough treatment of parabolic bundles can be found in Iyer and Simpson [8]. Parabolic Chern character is also defined in [8] (but we will not use it here). We adopt partially the notation of these articles.

We will discuss only parabolic bundles on complex surfaces. A good reference for usual two-dimensional bundles on surfaces is Friedman [4].

Definition 6.1 Let $X$ be a complex surface and $D$ be a simple normal crossing divisor with the irreducible decomposition $D=\bigcup_{i \in S} D_{i}$. A parabolic bundle $E_{*}$ on $X$ is given by a bundle $E$ with a collection of increasing filtrations by subsheaves $F_{a}^{i}$, indexed by $i \in S, a \in] 0,1]$ and satisfying the following properties:

(1) Every subsheaf $F_{a}^{i}$ is locally free.

(2) $E\left(-D_{i}\right) \subset F_{a}^{i}(S)$ for any $\left.\left.a \in\right] 0,1\right]$.

(3) The sets $\left\{a \mid F_{a}^{i}(E) / F_{<a}^{i}(E) \neq 0\right\}$ are finite for any $i$ in $S$.

Remark 6.2 A parabolic bundle $E_{*}$ on a complex surface $X$ with a simple normal crossing divisor $D$ induces natural filtrations on the restrictions $\left.E\right|_{D_{i}}$ by their vector subbundles. These filtrations are indexed by $a \in] 0,1]$ and defined by the formula:

$$
F_{a}^{i} /\left.E(-D) \subset E\right|_{D_{i}}
$$

Parabolic structure can be reconstructed from these filtrations (see Iyer and Simpson [8]).

Definition 6.3 The parabolic first Chern class of a parabolic bundle $E_{*}$ is given by the following formula:

$$
\operatorname{par}-\operatorname{ch}_{1}\left(E_{*}\right)=\operatorname{ch}_{1}(E)-\sum_{i} \sum_{a_{i}} a_{i} \cdot \operatorname{rank}_{D_{i}}\left(F_{a_{i}}^{i} / F_{<a_{i}}^{i}\right) \cdot\left[D_{i}\right] .
$$


Let $L$ be an ample line bundle on $S$. Then the parabolic degree of $E_{*}$ with respect to $L$ is given by

$$
\operatorname{pardeg}_{L}\left(E_{*}\right)=\int_{S} \operatorname{par}^{-c_{1}}\left(E_{*}\right) \cdot c_{1}(L)
$$

The parabolic second Chern characteristic number Let $(S, D)$ be a complex surface with a normal crossing divisor $D=\bigcup_{i=1}^{n} D_{i}$. Let $E_{*}$ be a parabolic vector bundle. We will recall now the formula for the parabolic second character of $E_{*}$ given by Mochizuki in [13]. His formula works in much larger generality but we need only the case of surfaces.

The parabolic second Chern character of $E_{*}$ is given as a sum of $c_{2}(E)$, the contributions of the divisors $D_{i}$, and the points of their intersections $D_{i} \cap D_{j}$.

To define the contributions of the points in $D_{i} \cap D_{j}$, for every $\left.\left.a_{i}, a_{j} \in\right] 0,1\right]$, consider the skyscraper sheaf $\mathrm{Gr}_{\left(a_{i}, a_{j}\right)}^{F}$ :

$$
F_{a_{i}}^{i} \cap F_{a_{j}}^{j} /\left(\left(F_{a_{i}}^{i} \cap F_{<a_{j}}^{j}\right)+\left(F_{<a_{i}}^{i} \cap F_{a_{j}}^{j}\right)\right) .
$$

This sheaf is supported at the points in $D_{i} \cap D_{j}$, and it is nontrivial only for finite set of $\left(a_{i}, a_{j}\right)$. Consider the sum

$$
v(i, j)=\sum_{p \in D_{i} \cap D_{j} ; a_{i}, a_{j}} a_{i} \cdot a_{j} \cdot \operatorname{rank}_{\mathrm{p}}\left(\operatorname{Gr}_{\left(a_{i}, a_{j}\right)}^{F}\right), \quad(v(i, j)=v(j, i)) .
$$

Definition 6.4 The second parabolic Chern character of the parabolic bundle $E_{*}$ is given by the formula

$$
\begin{aligned}
\operatorname{par}-\operatorname{ch}_{2}\left(E_{*}\right)=\operatorname{ch}_{2}(E)-\sum_{i ; a_{i}} a_{i} \cdot c_{1}\left(F_{a_{i}}^{i} / F_{<a_{i}}^{i}\right) & \\
& +\sum_{i ; a_{i}} \frac{1}{2} a_{i}^{2} \operatorname{rank}_{D_{i}}\left(F_{a_{i}}^{i} / F_{<a_{i}}^{i}\right) \cdot\left[D_{i} \cdot D_{i}\right]+\frac{1}{2} \sum_{i \neq j} v(i, j) .
\end{aligned}
$$

Stable bundles and Bogomolov-Gieseker inequality Here again we consider a surface $S$ with a parabolic bundle $E_{*}$. For any subsheaf $V$ of $E$ the filtration on $E$ induces a structure of a parabolic sheaf on $V$. Recall that a subsheaf $V$ of $E$ is called saturated is the quotient $E / V$ is torsion-free.

Definition 6.5 Let $L$ be an ample bundle on $S$. The bundle $E_{*}$ is called $\mu_{L}-$ stable (or slope stable) if for every saturated subsheaf $V$ of $E$,

$$
\operatorname{pardeg}_{L} V_{*}<\operatorname{pardeg}_{L} E_{*} .
$$


Remark 6.6 When $E$ is a rank 2 bundle in order to check its stability it is sufficient to consider only saturated locally free rank one subsheaves of $E$. Following [4] we call such subsheaves of $E$ sub-line bundles.

The following inequality (called Bogomolov-Gieseker inequality) is proven in [13] and was also proven in different terms in [12].

Theorem 6.7 Let $E_{*}$ be a $\mu_{L}$-stable parabolic bundle on the surface $S$. Then following inequality holds:

$$
\operatorname{par}-\operatorname{ch}_{2}\left(E_{*}\right)-\frac{1}{2} \operatorname{par}^{-\operatorname{ch}_{1}^{2}}\left(E_{*}\right) \leq 0 .
$$

Definition 6.8 Let $\left(X, D, E_{*}\right)$ be a complex surface with a simple normal crossing divisor $D$ with a parabolic bundle $E_{*}$, and let $\nabla$ be a unitary flat logarithmic connection on $E$ with poles at $D$. We say that $\nabla$ is compatible with $E_{*}$ if the following conditions hold.

(1) For every $i$ and $a \in] 0,1]$ the subbundle $F_{a}^{i} / E\left(-D_{i}\right)$ of $\left.E\right|_{D_{i}}$ is preserved by the residue map $\operatorname{Res}_{D_{i}}(\nabla)$.

(2) The eigenvalue of $\operatorname{Res}_{D_{i}}(\nabla)$ on the bundle $F_{a}^{i} / F_{<a}^{i}$ equals $-a$ (recall that this bundle is nontrivial only for finite number of values of $a$ ).

(3) The connection induced by $\nabla$ on $F_{a}^{i}\left(X \backslash D_{i}\right)$ extends to a logarithmic connection on $F_{a}^{i}(X)$.

Finally we can formulate the version of parabolic Kobayashi-Hitchin correspondence that we use later.

Theorem 6.9 Let $\left(X, D, E_{*}\right)$ be a complex projective surface with a simple normal crossing divisor $D$ and a parabolic bundle $E_{*}$. Suppose that $E_{*}$ is $\mu_{L}$-stable (with $L$ ample), has zero parabolic degree, and has zero second parabolic Chern number. Then there exists a flat unitary logarithmic connection on $E$ compatible with $E_{*}$.

This statement can be deduced from Mochizuki [13; 14] (see also Li [12]). We explain this very briefly skipping all details. In [13] Mochizuki works with parabolic Higgs bundles and proves Kobayashi-Hitchin correspondence for stable Higgs bundles with vanishing first and second Chern characters. The case that we are interested in is the particular case when the Higgs field is zero. So by [13] there exists a unitary flat metric on $E(X \backslash D)$ adapted to the parabolic structure. It follows further from [14] that the flat unitary connection corresponding to the flat metric constructed on $E(X \backslash D)$ extends to a logarithmic connection on $E$ and moreover this connection is compatible with the parabolic structure $E_{*}$. 


\section{Theorem of existence}

In this section we prove Theorem 1.12.

\subsection{A description of the proof}

Let $\left(L_{j}, \beta_{j}\right)$ be a weighted arrangement of lines in $\mathbb{C} P^{2}$. Recall that by $x_{i}$ we denote the multiple points of the arrangement of multiplicity at least $3 ; d_{i j}=1$ if $x_{i}$ belongs to $L_{j}$ and $d_{i j}=0$ otherwise. Suppose that $\left(L_{j}, \beta_{j}\right)$ satisfies the three conditions of Theorem 1.12.

To prove Theorem 1.12 we make the blow up $\pi: S \rightarrow \mathbb{C} P^{2}$ of $\mathbb{C} P^{2}$ at the points $x_{i}$ and consider on $S$ the pull back $E$ of the tangent bundle $E=\pi^{*} T \mathbb{C} P^{2}$. Using the weights $\beta_{j}$ we define a parabolic structure on $E$. We get a parabolic bundle $E_{*}$ with zero parabolic first Chern class and prove that $E_{*}$ is stable for a certain polarisation. Inequality (1-5) is just the Bogomolov-Gieseker inequality (Theorem 6.7).

In the case when the second parabolic Chern class of $E_{*}$ equals 0 using Theorem 6.9 we prove that there exists a logarithmic flat unitary connection on $T \mathbb{C} P^{2}$ and combining this with results of Section 4 we conclude the proof of Theorem 1.12.

\subsection{The parabolic bundle on the blown up $\mathbb{C} P^{2}$}

Let $\left(L_{j}, \beta_{j}\right)$ be an arrangement of lines in $\mathbb{C} P^{2}$, satisfying the inequalities (1-4). In this subsection we construct the parabolic structure on a 2-bundle on the blowup of $\mathbb{C} P^{2}$ and we calculate its parabolic Chern character. Let us fix some notation.

Notation Denote by $S$ the blow up of $\mathbb{C} P^{2}$ at the points $x_{1}, \ldots, x_{k}$ of multiplicity at least 3 and let $\pi: S \rightarrow \mathbb{C} P^{2}$ be the corresponding projection map.

Denote by $E$ the pullback bundle $\pi^{*} T \mathbb{C} P^{2}$.

For $j \in\{1, \ldots, n\}$ denote by $D_{j}$ the proper transform of $L_{j}$.

For $j \in\{n+1, \ldots, n+k\}$ denote by $D_{j}$ the exceptional line $P_{j-n}$ :

$$
D_{j}=P_{j-n}, \quad \pi\left(P_{j-n}\right)=x_{j-n} .
$$

The parabolic structure on the bundle $(S, E)$ For any $j \in\{1, \ldots, n\}$ the restriction of $E$ to $D_{j}$ contains a distinguished rank 1 subbundle - the pullback $\pi^{*} T L_{j}$ of the tangent bundle of $L_{j}$. Denote by $E_{j}$ the subsheaf of $E$, generated by the sections that 
are contained in $\pi^{*} T L_{j}$, being restricted to $D_{j}$. This subsheaf fits into the following exact sequence:

$$
\left.0 \rightarrow E_{j} \rightarrow E \rightarrow E\right|_{D_{j}} / \pi^{*} T L_{j} \rightarrow 0 .
$$

Now, for $1 \leq j \leq n$ we put

$$
F_{a}^{j}=E^{j} \quad \text { for } 0<a<1-\beta_{j}, \quad F_{a}^{j}=E \quad \text { for } 1-\beta_{j} \leq a \leq 1,
$$

and for $0<i \leq k$

$$
F_{a}^{n+i}=E\left(-D_{n+i}\right) \quad \text { for } 0<a<1-\alpha_{i}, \quad F_{a}^{n+i}=E \quad \text { for } 1-\alpha_{i} \leq a \leq 1 .
$$

Here, $1-\alpha_{i}=\frac{1}{2} \sum_{j} d_{i j}\left(1-\beta_{j}\right)$.

Proposition 7.1 The first and second parabolic Chern characters of $\left(E_{*}\right)$ are given by the following formulas:

$$
\begin{aligned}
& \operatorname{par}-\operatorname{ch}_{1}\left(E_{*}\right)=\operatorname{ch}_{1}(E)-\sum_{j=1}^{n}\left(1-\beta_{j}\right) D_{j}-2 \sum_{i=1}^{k}\left(1-\alpha_{i}\right) D_{n+i},
\end{aligned}
$$

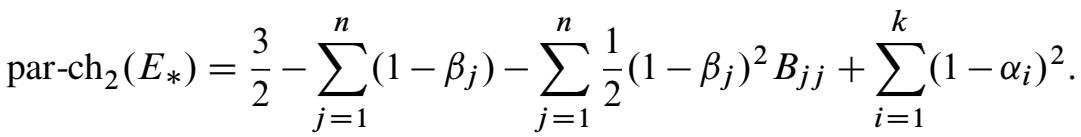

Proof The calculation of par- $\mathrm{ch}_{1}\left(\mathrm{E}_{*}\right)$ is a straight-forward application of Definition 6.3. For $j \in\{1, \ldots, n\}$ the quotient sheaf $F_{a}^{j} / F_{<a}^{j}$ has rank 1 for $a=1-\beta_{j}$ and is trivial otherwise. For $j \in\{n+1, \ldots, n+k\}$ the sheaf $F_{a}^{j} / F_{<a}^{j}$ has rank 2 for $a=1-\alpha_{j}$ and is trivial otherwise.

In order to calculate par-ch ${ }_{2}\left(\mathrm{E}_{*}\right)$ we need the following lemma.

Lemma 7.2 (1) For $1 \leq j_{1}<j_{2} \leq n$ we have $v\left(j_{1}, j_{2}\right)=0$.

(2) For $1<i_{1}<i_{2} \leq k$ we have $v\left(n+i_{1}, n+i_{2}\right)=0$.

(3) For $1 \leq j \leq n, 1 \leq i \leq k$ we have $v(j, n+i)=d_{i j}\left(1-\beta_{j}\right)\left(1-\alpha_{j}\right)$.

Proof (1) The sheaf $\operatorname{Gr}_{\left(a_{j_{1}}, a_{j_{2}}\right)}^{F}$ can be nontrivial only when $a_{j_{1}}=1-\beta_{j_{1}}$ and $a_{j_{2}}=1-\beta_{j_{2}}$. But in this case by construction the sheaf is equal to $E /\left(E_{j_{1}}+E_{j_{2}}\right)$. At the same time $E_{j_{1}}+E_{j_{2}}=E$.

(2) $\operatorname{Gr}_{\left(a_{n+i_{1}}, a_{n+i_{2}}\right)}^{F}$ is trivial because $D_{n+i_{1}} \cap D_{n+i_{2}}=\varnothing$.

(3) The sheaf $\operatorname{Gr}_{\left(a_{j}, a_{n+i}\right)}^{F}$ is nontrivial only when $a_{j}=1-\beta_{j}, a_{n+i}=1-\alpha_{i}$. In this case it is equal to $E /\left(E\left(-D_{n+i}\right) \cup E_{j}\right)$. It is supported at the points $D_{j} \cap D_{n+i}$ and has rank 1 at each point. 
Calculation of par-ch ${ }_{2}\left(E_{*}\right)$ According to Definition 6.4 we have

$$
\begin{aligned}
\operatorname{par}-\mathrm{ch}_{2}\left(E_{*}\right)=\frac{3}{2}-\sum_{j=1}^{n}\left(1-\beta_{j}\right) & -\sum_{j=1}^{n} \frac{1}{2}\left(1-\beta_{j}\right)^{2} B_{j j} \\
& -\sum_{i=1}^{k}\left(1-\alpha_{i}\right)^{2}+\sum_{i=1, j=1}^{k, n} d_{i j}\left(1-\beta_{j}\right)\left(1-\alpha_{i}\right) .
\end{aligned}
$$

Here we use the following facts:

(1) For $j \in(1, \ldots, n)$ we have $c_{1}\left(E / E_{j}\right)=1,\left[D_{j} \cdot D_{j}\right]=-B_{j j}$.

(2) For $i \in(1, \ldots, k)$ we have $E \mid D_{n+i}$ is trivial, $\left[D_{n+i} \cdot D_{n+i}\right]=-1$.

Finally, by Theorem 1.9 (use $p=q=1$ ) the last term is equal to $2 \sum_{i=1}^{k}\left(1-\alpha_{i}\right)^{2}$. This concludes the proof.

\subsection{Theorem on stability and additional lemmas}

In this subsection we prove that the parabolic bundle $E_{*}$ constructed above is stable with respect to an appropriate polarisation on $S$. Take $N \in \mathbb{Z}_{+}$,

$$
N>\max \left\{\frac{k}{\min _{i} \alpha_{i}}, \frac{2 k}{\min _{j, k}\left(\beta_{j}+\beta_{k}\right)}, \frac{3 k}{1-\max _{j} \beta_{j}}\right\},
$$

and define the following line bundle:

$$
L_{N}=O_{S}\left(-\sum_{i=1}^{k} P_{i}\right) \otimes p^{*} O(N)
$$

Note, that $L_{N}$ is ample since $N>k$.

Theorem 7.3 The parabolic bundle $\left(E_{*}, S\right)$ is $\mu_{L_{N}}-$ stable.

Let us first give a plan of the proof. The parabolic degree of $E_{*}$ is zero, so we need to show that the parabolic degree of any saturated sub-line bundle $V$ of $E$ is negative. Every line bundle on $S$ is of the form $O_{S}\left(\sum_{i} d_{i} P_{i}\right) \otimes \pi^{*} O(d)$. For sub-line bundles of $E$ we have $d \leq 1$ and the constant $N$ is chosen in such a way that the degree of $V \subset E$ can be positive only for $d \geq 0$. Since the parabolic weights are in [0,1], pardeg $V_{*} \leq \operatorname{deg} V$, so we only need to consider line subbundles of $E$ with $d=1,0$. In the case $d=1$ the pushdown $\pi_{*} V$ is contained in a sub-line bundle of $T \mathbb{C} P^{2}$ generated by sections tangent to a pencil of lines. We prove that $\operatorname{pardeg}\left(V_{*}\right)$ is negative 
comparing the degree of $V$ with the parabolic contribution, coming from the behavior of the pencil of lines with respect to the line arrangement on $\mathbb{C} P^{2}$.

Let us introduce some notation. For a point $x$ in $\mathbb{C} P^{2}$ denote by $L_{x}$ the sub-line bundle of $T \mathbb{C} P^{2}$ generated by the sections tangent to the pencil of lines containing $x$. For a section $v$ of $T \mathbb{C} P^{2}$ with isolated zeros denote by $L_{v}$ the sub-line bundle of $T \mathbb{C} P^{2}$ generated by $v$. The following lemma is standard, we omit the proof.

Lemma 7.4 Sub-line bundles of $T \mathbb{C} P^{2}$ have degree at most 1. Every saturated sub-line bundle of $T \mathbb{C} P^{2}$ of degree 1 equals $L_{x}$ for some $x$. Every saturated sub-line bundle of $T \mathbb{C} P^{2}$ of degree 0 equals $L_{v}$ for some $v$.

Lemma 7.5 Let $L=O_{S}\left(\sum_{i=1}^{k} d_{i} P_{i}\right) \otimes \pi^{*}(O(d))$ be a saturated sub-line bundle of $E$. Then $d \leq 1$. Suppose $d=1$ or $d=0$.

(1) If $d=1$ then $\pi_{*}(V) \subset L_{x}$ for some $x \in \mathbb{C} P^{2}$.

(2) If $d=0$ then $\pi_{*}(V) \subset L_{v}$ for some vector filed $v$ with isolated zeros.

(3) $\pi_{*}(V)$ coincides with $L_{x}$ or $L_{v}$ outside of the set $\left\{x_{1}, \ldots, x_{k}\right\}$.

Proof Consider $\left(\pi_{*} V\right)^{\vee \vee}$, this is a saturated sub-line bundle of $T \mathbb{C} P^{2}$. Its degree equals to $d$, so $d \leq 1$. In the case $d=1$ by the previous lemma $\left(\pi_{*} V\right)^{\vee \vee}$ is $L_{x}$ for some $x$, and if $d=0$ it is $L_{v}$ for some $v$. The sheaf $\pi_{*} V$ is a subsheaf of $\left(\pi_{*} V\right)^{\vee \vee}$ and it differs from it only at points $x_{i}$ for which $d_{i}<0$.

Lemma 7.6 Let $V=O_{S}\left(\sum_{i=1}^{k} d_{i} P_{i}\right) \otimes \pi^{*}(O(d))$ be a sub-line bundle of $E$. Then for any $i$ we have $d_{i} \leq 2-d$. In particular we have an upper bound on degree of $V$ :

$$
\operatorname{deg}_{L_{N}}(V)=c_{1}(V) \cdot c_{1}\left(L_{N}\right) \leq(2-d) k+N d .
$$

Proof Let us prove that for any $i$ it holds $d_{i} \leq 2-d$. For any line $P$ in $\mathbb{C} P^{2}$ we have $T \mathbb{C} P_{\mid P}^{2} \simeq O(1) \oplus O(2)$. Take a line $P$ that contains a point $x_{i}$ and doesn't contain any point $x_{j}$ for $j \neq i$. Let $P^{\prime}$ be the proper transform of $P$. Then again $E_{\mid P^{\prime}} \simeq O(1) \oplus O(2)$. Since $\operatorname{Hom}_{\mathrm{O}_{\mathrm{S}}}(V, E) \neq 0$, there is a line $P$ through $x_{i}$ for which Hom $_{\mathrm{O}^{\prime}}\left(V_{\mid P^{\prime}}, E_{\mid P^{\prime}}\right) \neq 0$. At the same time, by definition of $V$

$$
V_{\mid P^{\prime}}=O\left(d \pi^{*} H+d_{i} P_{i}\right)_{\mid P^{\prime}}=O\left(d+d_{i}\right) .
$$

It follows that

$$
\left(d+d_{i}\right) \leq 2
$$

Now we conclude the proof:

$$
\operatorname{deg}_{L_{N}}\left(O_{S}\left(\sum_{i} d_{i} P_{i}+d \pi^{*} H\right)\right)=\sum_{i} d_{i}+N d \leq(2-d) k+N d .
$$


Lemma 7.7 Let $\left(L_{j}, \beta_{j}\right)$ be a weighted line arrangement satisfying the conditions (1-4) of Theorem 1.12.

(1) For any point $x$ in $\mathbb{C} P^{2}$ the following inequality holds:

$$
\sum_{j \mid x \notin L_{j}}\left(1-\beta_{j}\right)>1+\frac{2 k}{N} .
$$

(2) A holomorphic vector field $v$ with isolated zeros can be tangent to at most 3 lines of the arrangement.

Proof (1) It is clear that the sum attains its minimum for a point that is a multiple point of the arrangement. Since $\sum_{j}\left(1-\beta_{j}\right)=3$, for a double point of the arrangement the sum in (7-4) is at least $1+\min _{j, k}\left(\beta_{j}+\beta_{k}\right)$. For a point $x_{i}$ of multiplicity more than 2 we have:

$$
\sum_{j \mid x \notin L_{j}}\left(1-\beta_{j}\right)=3-\sum_{j} d_{i j}\left(1-\beta_{j}\right)=3-2\left(1-\alpha_{i}\right) \geq 1+2 \min _{i}\left(\alpha_{i}\right) .
$$

(2) This is standard, the field $v$ has 3 zeros and it is tangent only to the lines that join these zeros.

\subsection{Proof of stability}

Proof of Theorem 7.3 By Lemma 7.5 and Lemma 7.6 any sub-line bundle $V$ of $E$ is of the form $O_{S}\left(\sum_{i=1}^{k} d_{i} P_{i}\right) \otimes \pi^{*}(O(d))$ with $d \leq 1$, and $\operatorname{deg}_{L_{N}}(V)<0$ if $d<0$. Since in our situation the parabolic weights are contained in $] 0,1]$, we have an inequality $\operatorname{pardeg}_{L_{N}}\left(V_{*}\right) \leq \operatorname{deg}_{L_{N}}(V)$. So it is necessary only to consider the cases when $d=1$ and $d=0$.

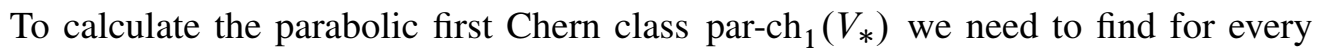
$j \in\{1, \ldots, n+k\}$ and $a \in] 0,1]$ the rank of the following quotient sheaf supported on $D_{j}$ :

$$
\left(V \cap F_{a_{j}}^{j}\right) /\left(V \cap F_{<a_{j}}^{j}\right) .
$$

Consider the case $d=1$. Then according to Lemma 7.5 there exists $x \in \mathbb{C} P^{2}$ such that $\pi_{*}(V) \subset L_{x}$.

In the case $j \in\{1, \ldots, n\}$ there are two possibilities.

If $x \in L_{j}$ then $V \subset F_{a_{j}}^{j}$ for all $0<a_{j} \leq 1$ so the corresponding quotient sheaf is always trivial. If $x \notin L_{j}$ then the quotient sheaf is nontrivial for $a_{j}=1-\beta_{j}$ and has rank one (this follows from Lemma 7.5). 
In the case $j \in\{n+1, \ldots, n+k\}$ the quotient sheaf is nontrivial for $a_{j}=1-\alpha_{j-n}$ and has rank 1 . This gives us the formula

$$
\operatorname{par}^{-\operatorname{ch}_{1}}\left(V_{*}\right)=\operatorname{ch}_{1}(V)-\sum_{j \mid x \notin L_{j}}\left(1-\beta_{j}\right) D_{j}-\sum_{i=1}^{n}\left(1-\alpha_{i}\right) D_{n+i} .
$$

We have the following sequence of inequalities

$$
\operatorname{pardeg}_{L_{N}}\left(V_{*}\right)=\operatorname{deg}_{L_{N}}(V)-c_{1}\left(L_{N}\right) \cdot\left(\sum_{j \mid x \notin L_{j}}\left(1-\beta_{j}\right) D_{j}+\sum_{i=1}^{n}\left(1-\alpha_{i}\right) D_{n+i}\right) .
$$

using Inequality (7-3) and the equality $2\left(1-\alpha_{i}\right)=D_{n+i} \cdot \sum_{j}\left(1-\beta_{j}\right) D_{j}$. This is less than or equal to

$$
N+k-N \sum_{j \mid x \notin L_{j}}\left(1-\beta_{j}\right)+\sum_{i} 2\left(1-\alpha_{i}\right)-\sum_{i}\left(1-\alpha_{i}\right) .
$$

using $0<\alpha_{i}<1$ and Inequality (7-4). Finally, this is less than

$$
N+2 k-N\left(1+\frac{2 k}{N}\right)=0 \text {. }
$$

The case $d=0$ is analogous. By Lemma 7.5 there exists a vector-field $v$ with isolated zeros such that $\pi_{*}(V) \subset L_{v}$. The arrangement contains more than 3 lines so there is at least one line that is not tangent to $v$. Making the same calculation as in the case $d=1$ and using $N>3 k /\left(1-\max _{j} \beta_{j}\right)$ we get

$$
\operatorname{pardeg}_{L_{N}}\left(V_{*}\right) \leq 2 k-N\left(1-\max _{j}\left(\beta_{j}\right)\right)+\sum_{i}\left(1-\alpha_{i}\right)<0 .
$$

Example 7.8 Consider the arrangement of 6 lines that pass through 4 generic points $x_{1}, \ldots, x_{4}$. For every $\left.\beta \in\right] 0,1\left[\right.$ we can associate the weight $\beta$ to the lines $x_{1} x_{i}$ and $1-\beta$ to the lines $x_{i} x_{j}, i, j>1$. We get a stable parabolic bundle on $\mathbb{C} P^{2}$ blown up at $x_{1}, \ldots, x_{4}$. When $\beta$ tends to 0 the parabolic degree of the sheaf corresponding to $L_{x_{1}}$ tends to zero and as a result for $\beta=0$ we don't get a PK metric on $\mathbb{C} P^{2}$.

\subsection{Proof of Theorem 1.12 and an application of the Bogomolov-Gieseker inequality}

Proof of Theorem 1.12 Let us sum up what we have done. We started with a weighted arrangement $\left(L_{j}, \beta_{j}\right)$ that satisfies the conditions of Theorem 1.12. We introduced a structure of parabolic bundle $E_{*}$ on the pullback $E$ of the tangent bundle $T \mathbb{C} P^{2}$ to the blow up of $\mathbb{C} P^{2}$. We proved that $E_{*}$ is stable (Theorem 7.3) and has zero first parabolic 
Chern class (Proposition 7.1). So inequality (1-5) follows from the calculation of second parabolic Chern number (Proposition 7.1) and Bogomolov-Gieseker inequality (Theorem 6.7). This proves the first part of the theorem.

If the equality is attained in (1-5) then the second parabolic Chern number of $E_{*}$ vanishes, so we can use parabolic Kobayashi-Hitchin correspondence (Theorem 6.9). Namely, there exists a unitary flat logarithmic connection on $E$ compatible with the parabolic structure of $E_{*}$. By Corollary 4.6 the constructed connection is torsion-free. Finally, using Theorem 4.13 we conclude that the corresponding flat connection on $T \mathbb{C} P^{2}$ defines a PK metric.

Let us give one corollary of Theorem 1.12. For a multiple point $x_{i}$ of a line arrangement $L_{1}, \ldots, L_{N}$ denote by $\mu_{i}$ the number of lines through $x_{i}$.

Corollary 7.9 Suppose that the multiplicity of every point of the arrangement $\left(L_{1}, \ldots, L_{N}\right)$ is less than $2 N / 3$. Then the following inequality holds:

$$
\sum_{i} \mu_{i} \geq \frac{N^{2}}{3}+N
$$

In the case of equality $N$ is divisible by 3 , every line intersects other lines in $N / 3+1$ points, and there is a PK metric on $\mathbb{C} P^{2}$ with conical angles $2 \pi(N-3) / N$ at the lines $L_{j}$.

Note that for a generic arrangement the total multiplicity is $N(N-1)$ while for the most degenerate arrangement it is $N$.

Proof We have the following equalities:

$$
\begin{aligned}
N^{2}=\left(\sum_{j} L_{j}\right)^{2} & =N+\sum_{j \neq k} L_{j} \cdot L_{k}=N+\sum_{i} \mu_{i}\left(\mu_{i}-1\right) \\
\sum_{i} \mu_{i}^{2} & =N^{2}-N+\sum_{i} \mu_{i}
\end{aligned}
$$

Associate to each $L_{j}$ weight $\beta_{j}=(N-3) / N$, then the arrangement satisfies the conditions of Theorem 1.12. Since all weights are equal we can treat double points of the arrangements as points of type $(1,1)$ and we get the following equalities:

$$
1-\alpha_{i}=\frac{3 \mu_{i}}{2 N}, \quad 1-\beta_{j}=\frac{3}{N}, \quad \sum_{j} B_{j j}=\sum_{i} \mu_{i}-N .
$$


Applying the Bogomolov-Gieseker inequality to the corresponding stable parabolic bundle $E_{*}$ and using (7-6) we get

$$
\begin{aligned}
& 0 \geq \operatorname{par}^{-c_{2}}\left(E_{*}\right)=\frac{\sum_{i} 9 \mu_{i}^{2}}{4 N^{2}}-\frac{9 \sum_{i} \mu_{i}-9 N}{2 N^{2}}-\frac{3}{2} \\
& =\frac{9 \sum_{i} \mu_{i}^{2}-18 \sum_{i} \mu_{i}+18 N-6 N^{2}}{4 N^{2}}=\frac{3 N^{2}+9 N-9 \sum_{i} \mu_{i}}{4 N^{2}} .
\end{aligned}
$$

Inequality (7-5) was proven previously by Langer [11] using different methods. We finish with the following lemma.

Lemma 7.10 For line arrangements satisfying condition of Theorem 1.12 the system of equations (1-1) from Theorem 1.11 (ie, $\sum_{k} B_{j k}\left(\beta_{k}-1\right)+1=0$ ) can be obtained by differentiating the Bogomolov-Gieseker inequality.

Proof Let $\left(L_{j}, \beta_{j}\right)$ be an arrangement satisfying conditions of Theorem 1.12. For a small deformation $\beta_{j}^{\prime}$ of $\beta_{j}$ the deformed parabolic bundle $E_{*}^{\prime}$ (defined in the same way as $E_{*}$ but using the weights $\beta_{j}^{\prime}$ instead $\beta_{j}$ ) is stable and satisfies the BogomolovGieseker inequality. The inequality is quadratic in $\beta_{j}$ and it attains its maxima (zero) for the initial data. So its derivatives in all directions vanish. This produces a system of linear equations on $\beta_{j}$. We will show that this system is equivalent to the system of equations (1-1) from Theorem 1.11. Consider the derivative

$$
\frac{\partial}{\partial \beta_{j}} \operatorname{par}^{-c_{2}}\left(E_{*}\right)-\frac{\partial}{\partial \beta_{j}} \frac{1}{2} \operatorname{par}-\operatorname{ch}_{1}^{2}\left(E_{*}\right) .
$$

Let us restrict this expression to the plane $\sum_{j}\left(\beta_{j}-1\right)=-3$. Then $\operatorname{par}^{-c_{1}}\left(E_{*}\right)=0$, and so the second term vanishes. $\operatorname{par}^{-\mathrm{ch}_{2}}\left(E_{*}\right)$ is given by Equation (7-2), and using $\left(1-\alpha_{i}\right)=\frac{1}{2} \sum_{k} d_{i k}\left(1-\beta_{k}\right)$ we get

$$
0=\frac{\partial}{\partial \beta_{j}}\left(\operatorname{par}_{-} \mathrm{ch}_{2}\left(E_{*}\right)\right)=1+\left(1-\beta_{j}\right) B_{j j}-\sum_{i} d_{i j}\left(1-\alpha_{i}\right) .
$$

Expanding $\left(1-\alpha_{i}\right)$, and using the definitions of $B_{j j}$ and $d_{i j}$ we rewrite the last term to obtain

$$
1+\left(1-\beta_{j}\right) B_{j j}-\frac{1}{2}\left(\left(B_{j j}+1\right)\left(1-\beta_{j}\right)+\sum_{k, i \mid d_{i j} d_{i k}=1}\left(1-\beta_{k}\right)\right) .
$$

Now using $\sum_{j}\left(\beta_{j}-1\right)=-3$, this equals

$$
\frac{1}{2}\left(2+\left(1-\beta_{j}\right) B_{j j}-\left(3+\sum_{k \neq j}\left(\beta_{k}-1\right)\right)+\sum_{k, i \mid d_{i j} d_{i k}=1}\left(1-\beta_{k}\right)\right) .
$$


Finally using the definition of $B_{j k}$, this is

$$
\frac{1}{2}\left(-1+\sum_{k}\left(1-\beta_{k}\right) B_{j k}\right) \text {. }
$$

\section{Further results, questions and directions}

In a subsequent paper we will use Theorem 1.12 to construct several infinite families of aspherical complex surfaces. Some of these families of surfaces admit a metric of type CAT(0). In particular some smooth compact quotients of the complex ball admit a PK metric of type CAT(0), this answers a question of Gromov and Davis.

For every PK surface its PK metric induces a positive $(1,1)$ current on it, so it should not be difficult to prove that every PK surface is a Kähler surface (in principle, one should be able to smoothen a bit the PK metric to get a smooth Kähler metric on the surface). It should be possible to show that nonalgebraic $K 3$ surfaces don't admit a PK metric, but we don't know any obstruction for the existence of PK metrics on algebraic surfaces. At the same time the set of examples of PK surfaces that we have is rather limited.

We hope that Theorem 1.5 extends to higher dimensions. Namely, that for a polyhedral Kähler manifold of any dimension the complex structure on the complement of the metric singularities extends to a complex analytic structure on the whole manifold. In particular the metric singularities should not have odd (real) codimension and all singularities of even codimension should have holomorphic directions. Note that in higher dimensions we can obtain complex manifolds with singularities even if we start with a topological polyhedral Kähler manifold (ie, the link of every point is a topological sphere). Indeed, by Brieskorn the link of the hypersurfaces $z_{1}^{2}+z_{2}^{2}+z_{3}^{2}+z_{4}^{3}+z_{5}^{6 k-1}=0,1 \leq k \leq 28$ in $\mathbb{C}^{5}$ is $S^{7}$ with one of 28 smooth structures. At the same time these hypersurfaces have a PK metric, induced by an obvious degree $24(6 k-1)$ ramified cover of the hyperplane $\sum_{i} z_{i}=0$.

The notion of polyhedral Kähler manifolds can be generalised in several directions. A polyhedral affine structure on a manifold is a choice of a simplicial decomposition and an affine structure on the complement of codimension 2 faces that restricts to the standard affine structure on the interior of every face of the top dimension. We say that a manifold $M^{2 n}$ is polyhedral complex affine if the holonomy is contained in $\operatorname{GL}(n, \mathbb{C})$, and singular faces of codimension 2 at which the holonomy is trivial have holomorphic directions (cf Definition 1.1). For complex dimension 2 we expect to get a theory similar to the one developed in this article. It should be possible to classify the singularities of complex codimension 2 but the list will be longer. 
If the holonomy of a polyhedral affine manifold is contained in the symplectic group $\mathrm{SP}(2 n)$ we call the manifold polyhedral nearly symplectic. It is not hard to see that every symplectic manifold admits a polyhedral nearly symplectic structure. But the converse should be wrong already for 4-manifolds, so we adjust the definition.

Definition 8.1 Let $M^{4}$ be a polyhedral nearly symplectic manifold and let $M_{1}^{4}(\varepsilon)$ be a neighborhood of the union of all edges. The PL symplectic structure on $M^{4} \backslash M_{1}^{4}(\varepsilon)$ can be smoothen along the faces of codimension 2 to a genuine symplectic form $w$. Let $c_{1}$ be the first Chern class of an almost complex structure tamed by $w$ on $M_{1}^{4}(\varepsilon)$. We call $M^{4}$ polyhedral symplectic if for every surface $S$ contained in $M_{1}^{4}(2 \varepsilon) \backslash M_{1}^{4}(\varepsilon)$ we have $c_{1} \cdot S=0$.

It is not hard to prove that every symplectic 4-manifold admits a polyhedral symplectic structure. More importantly, we conjecture that every polyhedral symplectic 4-manifold admits a symplectic smoothing.

Another interesting direction to generalise PK manifolds is to consider complex manifolds with Kähler metric of constant holomorphic curvature and conical singularities at holomorphic geodesic divisors. In the case of negative curvature these manifolds will be generalisations of complex hyperbolic orbifolds, ie quotients of the complex ball $B^{n}$ by a lattice of $\mathrm{SU}(n, 1)$. These manifolds presumably are the same as Thurston $\left(\mathbb{C} H^{n}, \mathrm{SU}(n, 1)\right)$-cone manifolds [19]. In the case of surfaces it is sufficient to ask that singularities of the metric form a complex curve, at points that are not multiple there is a local isometric action of the group $\mathrm{SU}(1,1) \times S^{1}$, and at the multiple points there is an holomorphic isometric action of $\mathbb{R}^{1}$. It should be possible to generalise Theorem 1.12 to this setting using parabolic Kobayashi-Hitchin correspondence for parabolic Higgs bundles [13], and using ideas from [18]. It would be interesting to reprove (or even generalise) results of [3] using this approach.

Finally we hope to prove the following conjecture.

Conjecture 8.2 For every arrangement satisfying conditions of Theorem 1.12 its complement is of the type $K(\pi, 1)$.

The converse to this conjecture is wrong because Proposition 5.2 permits us to check that some simplicial arrangements are not PK. At the same time by a theorem of Deligne all (complexified) simplicial arrangement have a complement of the type $K(\pi, 1)$. 


\section{References}

[1] A Borel, P-P Grivel, B Kaup, A Haefliger, B Malgrange, F Ehlers, Algebraic Dmodules, Perspectives in Math. 2, Academic Press, Boston (1987) MR882000

[2] J Cheeger, A vanishing theorem for piecewise constant curvature spaces, from: "Curvature and topology of Riemannian manifolds (Katata, 1985)", (K Shiohama, T Sakai, T Sunada, editors), Lecture Notes in Math. 1201, Springer, Berlin (1986) 33-40 MR859575

[3] W Couwenberg, G Heckman, E Looijenga, Geometric structures on the complement of a projective arrangement, Publ. Math. Inst. Hautes Études Sci. (2005) 69-161 MR2217047

[4] R Friedman, Algebraic surfaces and holomorphic vector bundles, Universitext, Springer, New York (1998) MR1600388

[5] B Grünbaum, Arrangements of hyperplanes, from: "Proceedings of the Second Louisiana Conference on Combinatorics, Graph Theory and Computing (Louisiana State Univ., Baton Rouge, La., 1971)", (R C Mullin, K B Reid, D P Roselle, R S D Thomas, editors), Louisiana State Univ., Baton Rouge, La. (1971) 41-106 MR0320895

[6] B Grünbaum, Arrangements and spreads, Conf. Board of Math. Sci. Reg. Conf. Ser. in Math. 10, Amer. Math. Soc. (1972) MR0307027

[7] F Hirzebruch, Algebraic surfaces with extremal Chern numbers (based on a dissertation by T Höfer, Bonn, 1984), Uspekhi Mat. Nauk 40 (1985) 121-129 MR807793 Translated from the English by I A Skornyakov, International conference on current problems in algebra and analysis (Moscow-Leningrad, 1984)

[8] J N N Iyer, C Simpson, The Chern character of a parabolic bundle, and a parabolic Reznikov theorem in the case of finite order at infinity arXiv:math. AG/06121444

[9] J Kaneko, S Tokunaga, M Yoshida, Complex crystallographic groups. II, J. Math. Soc. Japan 34 (1982) 595-605 MR669270

[10] W Kühnel, T F Banchoff, The 9-vertex complex projective plane, Math. Intelligencer 5 (1983) 11-22 MR737686

[11] A Langer, Logarithmic orbifold Euler numbers of surfaces with applications, Proc. London Math. Soc. (3) 86 (2003) 358-396 MR1971155

[12] J Li, Hermitian-Einstein metrics and Chern number inequalities on parabolic stable bundles over Kähler manifolds, Comm. Anal. Geom. 8 (2000) 445-475 MR1775134

[13] T Mochizuki, Kobayashi-Hitchin correspondence for tame harmonic bundles and an application, Astérisque (2006) viii+117 MR2310103

[14] T Mochizuki, Asymptotic behaviour of tame harmonic bundles and an application to pure twistor D-modules. I, Mem. Amer. Math. Soc. 185 (2007) xii+324 MR2281877 
[15] M Ohtsuki, A residue formula for Chern classes associated with logarithmic connections, Tokyo J. Math. 5 (1982) 13-21 MR670900

[16] S Orshanskiy, A PL-manifold of nonnegative curvature homeomorphic to $S^{2} \times S^{2}$ is a direct metric product arXiv:0807.1922

[17] A Petrunin, Polyhedral approximations of Riemannian manifolds, Turkish J. Math. 27 (2003) 173-187 MR1975337

[18] C T Simpson, Constructing variations of Hodge structure using Yang-Mills theory and applications to uniformization, J. Amer. Math. Soc. 1 (1988) 867-918 MR944577

[19] W P Thurston, Shapes of polyhedra and triangulations of the sphere, from: "The Epstein birthday schrift”, (I Rivin, C Rourke, C Series, editors), Geom. Topol. Monogr. 1, Geom. Topol. Publ., Coventry (1998) 511-549 MR1668340

[20] M Troyanov, Les surfaces euclidiennes à singularités coniques, Enseign. Math. (2) 32 (1986) 79-94 MR850552

Department of Mathematics, Imperial College

London, SW7 2AZ, UK

dpanov@imperial.ac.uk

http://www.ma.ic.ac.uk/ dpanov

Proposed: Dmitri Burago

Received: 29 January 2009

Seconded: Simon Donaldson, Jim Bryan

Revised: 5 May 2009 